\title{
Total parasite biomass but not peripheral parasitaemia is associated with endothelial and haematological perturbations in Plasmodium vivax patients
}

João L Silva-Filho ${ }^{1,2 * \dagger}$, João CK Dos-Santos ${ }^{1,3 \dagger}$, Carla Judice ${ }^{1}$, Dario Beraldi ${ }^{2}$ Kannan Venugopal ${ }^{2}$, Diogenes Lima ${ }^{4 \ddagger}$, Helder I Nakaya ${ }^{4,5}$, Erich V De Paula ${ }^{6}$, Stefanie CP Lopes ${ }^{6,7,8}$, Marcus VG Lacerda ${ }^{7,8}$, Matthias Marti ${ }^{2 \star}$, Fabio TM Costa ${ }^{1 *}$

${ }^{1}$ Laboratory of Tropical Diseases - Prof. Luiz Jacintho da Silva, Department of Genetics, Evolution, Microbiology and Immunology, Institute of Biology, University of Campinas, Campinas, Brazil; ${ }^{2}$ Wellcome Centre for Integrative Parasitology, Institute of Infection, Immunity \& Inflammation, University of Glasgow, Glasgow, United Kingdom; ${ }^{3}$ Post-Graduation in Medical Pathophysiology, School of Medical Sciences, University of Campinas, Campinas, Brazil; ${ }^{4}$ School of Pharmaceutical Sciences, University of São Paulo, São Paulo, Brazil; ${ }^{5}$ Hospital Israelita Albert Einstein, São Paulo, Brazil; ${ }^{\circ}$ Department of Clinical Pathology, School of Medical Sciences, University of Campinas, Campinas, Brazil; ${ }^{7}$ Institute Leônidas \& Maria Deane, Fiocruz, Manaus, Brazil; ${ }^{8}$ Tropical Medicine Foundation Dr. Heitor Vieira Dourado, Manaus, Brazil
*For correspondence:

joao.dasilvafilho@glasgow.ac.uk (JLS-F);

matthias.marti@glasgow.ac.uk (MM);

fabiotmc72@gmail.com (FTMC)

'These authors contributed equally to this work

${ }^{\ddagger}$ Deceased

Competing interest: The authors declare that no competing interests exist.

Funding: See page 21

Preprinted: 20 March 2021

Received: 17 June 2021

Accepted: 28 September 2021

Published: 29 September 2021

Reviewing Editor: Urszula Krzych, Walter Reed Army Institute of Research, United States

(c) Copyright Silva-Filho et al. This article is distributed under the terms of the Creative Commons Attribution License, which permits unrestricted use and redistribution provided that the original author and source are credited.
Abstract Plasmodium vivax is the major cause of human malaria in the Americas. How P. vivax infection can lead to poor clinical outcomes, despite low peripheral parasitaemia, remains a matter of intense debate. Estimation of total P. vivax biomass based on circulating markers indicates existence of a predominant parasite population outside of circulation. In this study, we investigate associations between both peripheral and total parasite biomass and host response in vivax malaria. We analysed parasite and host signatures in a cohort of uncomplicated vivax malaria patients from Manaus, Brazil, combining clinical and parasite parameters, multiplexed analysis of host responses, and ex vivo assays. Patterns of clinical features, parasite burden, and host signatures measured in plasma across the patient cohort were highly heterogenous. Further data deconvolution revealed two patient clusters, here termed Vivax ${ }^{\text {low }}$ and Vivax ${ }^{\text {high }}$. These patient subgroups were defined based on differences in total parasite biomass but not peripheral parasitaemia. Overall Vivax ${ }^{\text {low }}$ patients clustered with healthy donors and Vivax ${ }^{\text {high }}$ patients showed more profound alterations in haematological parameters, endothelial cell (EC) activation, and glycocalyx breakdown and levels of cytokines regulating different haematopoiesis pathways compared to Vivax ${ }^{\text {low }}$. Vivax ${ }^{\text {high }}$ patients presented more severe thrombocytopenia and lymphopenia, along with enrichment of neutrophils in the peripheral blood and increased neutrophil-to-lymphocyte ratio (NLCR). When patients' signatures were combined, high association of total parasite biomass with a subset of markers of EC activation, thrombocytopenia, and lymphopenia severity was observed. Finally, machine learning models defined a combination of host parameters measured in the circulation that could predict the extent of parasite infection outside of circulation. Altogether, our data show that total parasite biomass is a better predictor of perturbations in host homeostasis in $P$. vivax patients than peripheral 
parasitaemia. This supports the emerging paradigm of a $P$. vivax tissue reservoir, particularly in the haematopoietic niche of bone marrow and spleen.

\section{Introduction}

Malaria remains a heavy burden across endemic regions worldwide. In 2018, Plasmodium vivax infection accounted for $41 \%$ of all malaria cases outside of Sub-Saharan Africa, with a total of 6.5 million cases and more than 2 billion people in 90 countries at risk (World Malaria Reports, 2019). There are concerns that $P$. vivax elimination will be significantly more difficult than $P$. falciparum as the current measures for malaria control are less effective for $P$. vivax than for Plasmodium falciparum, with the elimination of the former presenting a major challenge in areas that successfully reduced $P$. falciparum burden. This persistence is due to some unique biological features complicating treatment and elimination, including low peripheral parasitaemia and presence of dormant liver stages (hypnozoites) which relapse weeks or months after blood infection has been cleared.

$P$. vivax infection is associated with low peripheral parasitaemia $(<2 \%)$ as a result of a strict host cell tropism to immature reticulocytes (Malleret et al., 2015; Mayor and Alano, 2015) that are exceedingly rare in peripheral blood $(<2 \%)$ but highly prevalent in the haematopoietic niche of bone marrow (BM) and spleen (Klei et al., 2017; Rhodes et al., 2016). Because of limited microvascular adherence in vivo and endothelial cell (EC) binding in vitro (Lacerda et al., 2012; Valecha et al., 2009), it was generally assumed that peripheral parasitaemia reflects the majority of $P$. vivax parasites during infection. However, discrepancy of parasite biomass based on systemic biomarkers such as Plasmodium lactate dehydrogenase (pLDH) compared to peripheral parasitaemia supports existence of a major $P$. vivax reservoir outside of circulation, particularly in patients with complicated outcomes (Barber et al., 2015). In support of this hypothesis, studies have demonstrated that late asexual blood stage $P$. vivax parasites (i.e. schizonts) are capable of cytoadhering to endothelial host receptors (Carvalho et al., 2010; De las Salas et al., 2013) and present at reduced abundance compared to the other blood stages in the blood of P. vivax patients (Obaldia et al., 2018; Lopes et al., 2014). In experimentally infected non-human primates (NHPs), a significant enrichment of sexual stages (gametocytes) and schizonts in BM sinusoids and parenchyma has been observed (Obaldia et al., 2018), supporting previous evidence from multiple case reports that identified $P$. vivax in BM and spleen (Yx et al., 2009; Wickramasinghe et al., 1989; Wickramasinghe and Abdalla, 2000; Salutari et al., 1996; Baro et al., 2017; Machado Siqueira et al., 2012; Lacerda et al., 2008; Brito et al., 2020). A series of recent studies in acute and chronic human $P$. vivax infection have meanwhile provided direct evidence that BM and spleen represent the major reservoir of parasite biomass in $P$. vivax infection (Baro et al., 2017; Brito et al., 2020; Kho et al., 2021a; Kho et al., 2021b).

$P$. vivax parasites can elicit a potent host response, including inflammation and EC activation, and cause severe and fatal manifestations at significantly lower peripheral parasitaemia than the more virulent species, P. falciparum (Barber et al., 2015; Yeo et alı, 2010). However, the pathogenic mechanisms underlying these alterations in host homeostasis and their relationship with $P$. vivax biomass are not fully understood (Lacerda et al., 2011; Naing and Whittaker, 2018; Rodriguez-Morales et al., 2005; Tangpukdee et al., 2008).

Here we systematically investigated host responses in a cross-sectional cohort of uncomplicated $P$. vivax patients from Manaus, in the Brazilian Amazon region. Our analysis revealed an association between alterations in host homeostasis, including EC activation, damage, and haematological disturbances, such as thrombocytopenia, lymphopenia, and increased neutrophils turnover, with total parasite biomass but not peripheral parasitaemia. These findings are in line with the emerging paradigm of a clinically relevant parasite reservoir outside of circulation and merit systematic investigations of this reservoir in vivax malaria.

\section{Results}

\section{Uncomplicated $P$. vivax patients present with haematological changes}

We have conducted a cross-sectional study with uncomplicated $P$. vivax malaria patients seen at FMTHVD in Manaus, Brazil. We included 79 adult patients (median age of 36 years) with confirmed $P$. vivax infection (smear and PCR positive) and 34 age- and sex-matched uninfected healthy donors 
Table 1. Demographic, parasite, and multiplexed microbead-based immunoassay (Luminex) data obtained from the plasma of a representative subset of $31 \mathrm{P}$. vivax patients and 9 healthy donors (controls).

\begin{tabular}{|c|c|c|c|}
\hline Parameters & $\begin{array}{l}\text { Healthy donors }(n= \\
\text { 36) }\end{array}$ & $\begin{array}{l}\text { Symptomatic } \mathrm{Pv} \text { patients ( } \mathrm{n}= \\
\text { 79) }\end{array}$ & $\begin{array}{l}\text { p-Value } \\
\text { (Pv vs. control) }\end{array}$ \\
\hline & Median [IQ 25-75] & Median [IQ 25-75] & \\
\hline Age & $32(23-49)$ & $36(28-45)$ & 0.06 \\
\hline Parasitaemia (103 /mL) & - & $4.29[1.86-6.62]$ & \\
\hline Parasitaemia (\%) & - & $0.76[0.57-1.25]$ & \\
\hline $\begin{array}{l}\text { Parasite load (copies 18S RNA/ } \\
\mathrm{mL} \text { ) }\end{array}$ & - & $26,642[9253-522,297]$ & \\
\hline PvLDH (O.D.) & - & $0.18[0.005-0.34]$ & \\
\hline Plasma biomarkers & Healthy donors $(n=9)$ & Symptomatic Pv patients $(n=31)$ & $\begin{array}{l}\text { p-Value } \\
\text { (Pv vs. control) }\end{array}$ \\
\hline TNF- $\alpha(p g / m L)$ & $17.2[11.0-22.3]$ & $38.4[30.0-69.6]$ & $<0.0001$ \\
\hline $\mathrm{IL}-1 \alpha(\mathrm{pg} / \mathrm{mL})$ & $11.9[10.0-19.5]$ & 25.4 [19.8-33.5] & 0.0004 \\
\hline $\mathrm{IL}-1 \beta(\mathrm{pg} / \mathrm{mL})$ & $12.0[8.0-12.8]$ & $21.4[14.5-27.6]$ & $<0.0001$ \\
\hline IL-6 (pg/mL) & $3.0[2.5-3.7]$ & $33.4[7.6-133.1]$ & $<0.0001$ \\
\hline IL-8 (pg/mL) & $2.2[0.6-2.4]$ & $6.4[2.7-19.9]$ & 0.0005 \\
\hline IL-10 (pg/mL) & $-^{\star}$ & 314 [169-562] & - \\
\hline G-CSF $(\mathrm{pg} / \mathrm{mL})$ & 9.485 [9.485-9.485] & $101.5[33.49-239.6]$ & $<0.0001$ \\
\hline L-selectin (ng/mL) & 326 [287-391] & 481 [386-579] & 0.0019 \\
\hline ICAM-1 (ng/mL) & 323 [260-464] & 634 [456-849] & 0.0026 \\
\hline VCAM-1 (ng/mL) & 819 [623-959] & 2875 [1753-5108] & $<0.0001$ \\
\hline E-Selectin (ng/mL) & $26.4[22.5-33.7]$ & $56.7[41.5-74.1]$ & 0.0001 \\
\hline P-selectin (ng/mL) & $17.0[15.4-20.6]$ & $22.2[17.6-25.7]$ & 0.0621 \\
\hline Angiopoietin-1 (ng/mL) & $0.4[0.3-0.6]$ & $0.5[0.2-0.9]$ & 0.8874 \\
\hline Angiopoietin-2 (ng/mL) & $1.8[1.5-2.1]$ & $4.3[2.7-5.3]$ & 0.0003 \\
\hline Ang-2:Ang-1 ratio & $4.2[2.7-5.6]$ & $12.14[2.7-40.2]$ & 0.03 \\
\hline WWF-A2 (pg/mL) & 126 [120-150] & 218 [199-277] & $<0.0001$ \\
\hline ADAMTS13 (ng/mL) & 1110 [483-1740] & 776 [572-1328] & 0.5485 \\
\hline PAl-1 (pg/mL) & 78.9 [62.4-96.4] & 112 [69.3-242] & 0.1541 \\
\hline CD40L (ng/mL) & $0.5[0.4-0.7]$ & $1.0[0.7-1.3]$ & 0.0001 \\
\hline Syndecan-1 (ng/mL) & $1.8[1.6-2.4]$ & 3.7 [2.9-6.0] & 0.0003 \\
\hline IL-11 (ng/mL) & $3.5[2.9-4.3]$ & $5.7[4.7-6.4]$ & $<0.0001$ \\
\hline $\mathrm{TPO}(\mathrm{ng} / \mathrm{mL})$ & $2.0[1.7-2.2]$ & $3.0[2.6-3.4]$ & $<0.0001$ \\
\hline CXCL4 (ng/mL) & $0.8[0.6-1.2]$ & $1.4[0.7-2.8]$ & 0.1236 \\
\hline CXCL7 (ng/mL) & $0.4[0.4-0.5]$ & $0.73[0.4-1.7]$ & 0.1958 \\
\hline $\mathrm{SCF}(\mathrm{pg} / \mathrm{mL})$ & 47.61 [37.34-89.34] & 45.68 [36.22-61.39] & 0.1594 \\
\hline
\end{tabular}

PvLDH: P. vivax lactate dehydrogenase.

* $=$ under detection limit. 


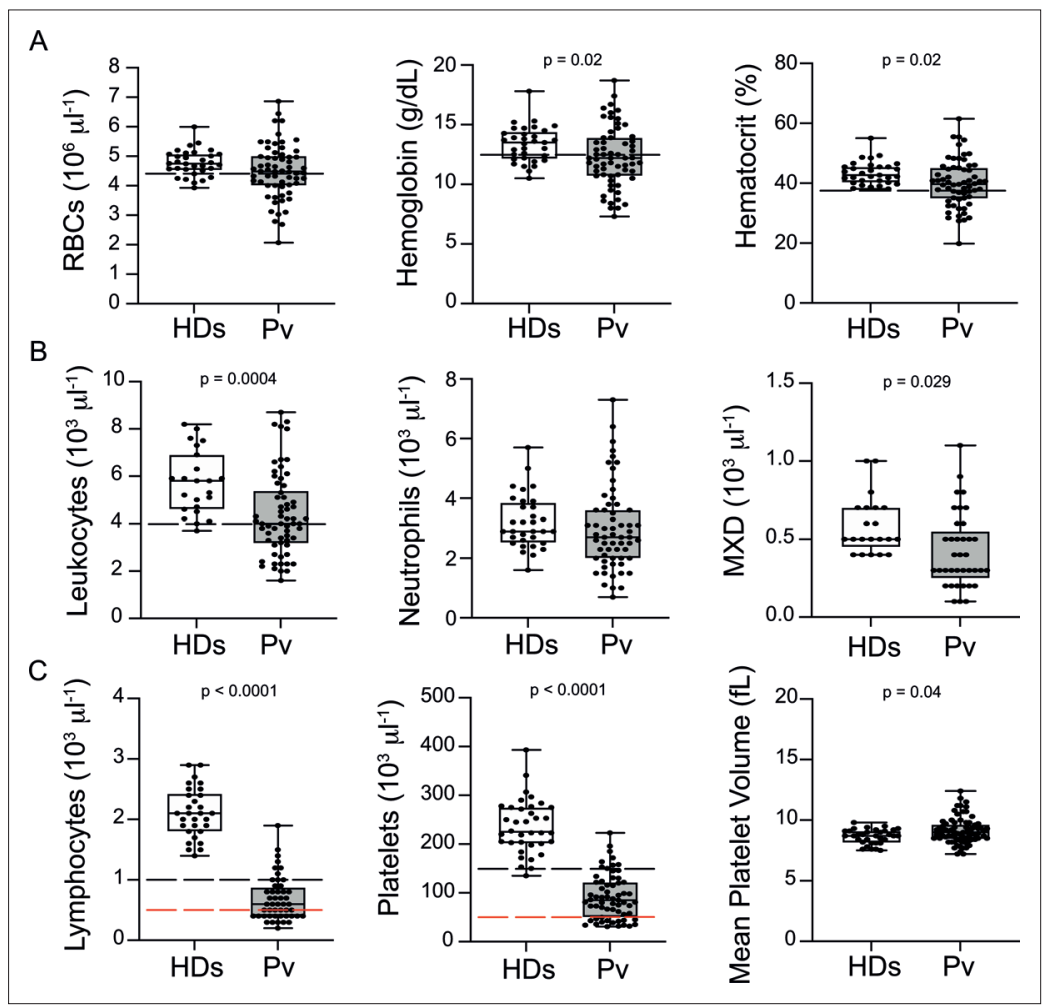

Figure 1. Clinical data of P. vivax patients (Pv) and healthy donors (HDs). (A) Red blood cell parameters. Shown are red blood cell counts, haemoglobin levels, and haematocrit. (B) Other blood cell parameters. Shown are numbers of leukocytes, neutrophils, and monocytes, basophils, and eosinophils (MXD). (C) Number of lymphocytes, platelets, and mean platelet volume (MPV). Parameters are depicted as box plots showing each individual value and the median with maximum and minimum values. Dashed lines in black mark the minimum threshold for normal reference values, while lines in red mark threshold for severe lymphopenia and thrombocytopenia, respectively. Two-tailed Student's t-test was used to compare variables with normally distributed data, and Mann-Whitney test was used to compare variables with non-normal distributions; $\mathrm{p}$-value is indicated above the graph when $\mathrm{p}<0.05$. HDs: healthy donors (controls, $n=34) ;$ Pv: P. vivax-infected patients $(n=79)$.

The online version of this article includes the following figure supplement(s) for figure 1:

Figure supplement 1. Demographic and clinical features of all $P$. vivax-infected patients compared with selected 31 patients for multiplex bead-based assay and downstream analysis.

(controls; Table 1). All individuals within the study including controls were from the state of Amazonas, in the Amazon region of Brazil. Blood was collected at enrolment for determination of haematological parameters, peripheral parasitaemia by Giemsa staining of blood smears, and PCR to determine genome copy numbers. Preparation of poor platelet plasma (PPP) was done within $15 \mathrm{~min}$ of sampling. The median peripheral parasitaemia was 4290 infected red blood cells (iRBCs)/ $\mu \mathrm{L}$ of blood (25-75 interquartile range 1860-6620 parasites/ $\mu \mathrm{L}$ ) and parasite load of 26,642 copies of $18 \mathrm{~S}$ RNA/ $\mu \mathrm{L}(25-75$ interquartile range 9253-522,297). We also measured total parasite biomass independently of peripheral parasitaemia by quantifying levels of $P$. vivax lactate dehydrogenase ( $P v L D H)$ in plasma (Table 1).

Analysis of haematological parameters revealed significantly reduced haemoglobin levels and haematocrit across $P$. vivax patients compared to controls, with anaemia in $38 \%$ of the patients (Figure 1A). Similarly, leukocyte numbers were significantly decreased (mean \pm SD: $4.36 \pm 1.74 \times 10^{3}$ / $\mu \mathrm{L}$ vs. $5.72 \pm 1.34 \times 10^{3} / \mu \mathrm{L}, \mathrm{p}=0.0004$ ), with $54.5 \%$ of the patients presenting with leukopenia (defined as a leukocyte count $<4000$ cells $/ \mu \mathrm{L})$. In contrast, neutrophil counts were not significantly different, and only $8.3 \%$ of $P$. vivax patients were presenting with neutropenia (neutrophil counts $<1500$ cells/ $\mu \mathrm{L}$ ) (Figure 1B). Other myeloid cell populations, however, such as monocytes, basophils, and eosinophils (MXD), were significantly reduced. We also observed a significant reduction in lymphocyte and platelet counts in this cohort (Figure 1C), with $80 \%$ presenting with lymphopenia (lymphocyte 
counts $<1000$ cells $/ \mu \mathrm{L}$ ) and $87 \%$ with thrombocytopenia (platelet counts $<150,000$ cells $/ \mu \mathrm{L}$ ), many of them with severely reduced levels (Figure 1C). Alterations in platelet counts were accompanied by the release of mega platelets in the peripheral circulation as a significant increase on mean platelet volume was observed (Figure $1 \mathrm{C}$ ).

In summary, patients in our cohort presented with a wide range of parasitaemia and uncomplicated clinical signs of $P$. vivax infection at medical consultation. However, significant haematological abnormalities were present in the majority of patients during early onset of disease, in line with previous findings (Barber et al., 2015; Lacerda et al., 2011; de Mast et al., 2009; de Mast et al., 2007; Gomes et al., 2014; Park et al., 2003; Punnath et al., 2019).

\section{Unsupervised clustering reveals two $P$. vivax patient subgroups that differ in parasite biomass: Vivax ${ }^{\text {high }}$ vs. Vivax low}

To determine whether the observed changes were associated with specific host signatures, particularly circulating biomarkers of haematological and endothelial changes, we applied a multiplexed microbead-based immunoassay (Luminex) in a representative subset of $31 P$. vivax patients and 9 controls, as explained in the Materials and methods section (Figure 1-figure supplement 1). We selected a series of circulating biomarkers associated with haematological changes, including cytokines altering thrombopoiesis (TPO and IL-11), myelopoiesis, and lymphopoiesis (TNF- $\alpha$, IL-1 $\alpha$, IL-1 $\beta$, IL-6, IL-8, G-CSF) (Boiko and Borghesi, 2012; Chiba et al., 2018; Kovtonyuk et al., 2016). In addition, we selected markers of EC and platelet activation, coagulation (ICAM-1, VCAM-1, E-selectin, P-selectin, Angiopoietin-1 and -2, CD40L, VWF-A2, ADAMTS13, PAI-1, CXCL4, CXCL7), and EC glycocalyx breakdown (Syndecan-1).

We observed significant upregulation of multiple cytokines associated with haematological changes in the $P$. vivax patients compared to control (Table 1). In addition, patient samples exhibited a strong phenotype of increased EC activation, glycocalyx breakdown and coagulation. The high interquartile range in parasitaemia and host signatures (Table 1) suggested a heterogenous phenotype across the patient population. In order to identify possible stratification of patients into distinct subgroups, we further analysed the clinical data (Figure 1), parasite parameters, and Luminex data (Table 1) from the same $31 \mathrm{P}$. vivax patients and 9 controls as above. After z-score normalization, principal component analysis (PCA) was performed for data dimensionality reduction, considering the large number of variables in our dataset. Next, we ran K-means clustering ( $k$ ) followed by bootstrapping (Figure 2A and B, Figure 2-figure supplement 1, Figure 2-figure supplement 2, Figure 2-figure supplement 2-source data 1) to identify possible subclusters of individuals. This analysis revealed consistent separation of samples into two clusters, one of them including all controls (cluster $1 \mathrm{a}$ ) and a subset of 14 patient samples (cluster $1 \mathrm{~b}$ ) and a second one representing the remaining 17 patient samples (cluster 2) (Figure $\mathbf{2 A}$ and B). In order to visualize covariables of the observed patient distribution (PCA) and clustering (K-means), we plotted the correlation (loading score) of each input variable with a principal component (PC; Figure 2C, Figure 2-source data 1). This analysis demonstrated covariation of lymphopenia and thrombocytopenia, on the one hand, and markers of EC changes, platelet production, activation, and parasite parameters (PvLDH and peripheral parasitaemia), on the other hand, as major contributors to the PCs (Figure 2C). Direct comparison of the two patient subgroups revealed significant higher total parasite biomass but not peripheral parasitaemia or parasite load (Figure 3A). In agreement with previous findings (Barber et al., 2015; Fonseca et al., 2017; Silva-Filho et al., 2021), z-score comparison further demonstrated that total parasite biomass was higher than and not correlated with peripheral parasitaemia levels or parasite load, particularly in patients of cluster 2 (Figure 3B and C). In addition, PvLDH was the input parasite variable with the highest loading score (correlation $=0.59$ ) and lowest $p$-value (0.0000917) in the first PC dimension when compared with peripheral parasitaemia and parasite load (Figure 2C, Figure 2-source data 1). Indeed, using a best-fit classification tree model and a random forest machine learning model defining K-means clusters as categorical outcome, PvLDH is the best parasite predictor attribute segregating patients into clusters $1 \mathrm{~b}$ and 2 (Figure 3D and E). After both models were trained in a set of 30 individuals, randomly selected by the training algorithm set, they were tested in the 10 remaining individuals, where all cluster $1 \mathrm{a}$ (control) individuals and $80 \%$ of $P$. vivax patients were correctly classified into either cluster $1 \mathrm{~b}$ or cluster 2 . Based on these observations, we designated cluster $1 \mathrm{a}$ as Control cluster (representing the healthy donors), cluster $1 \mathrm{~b}$ as 


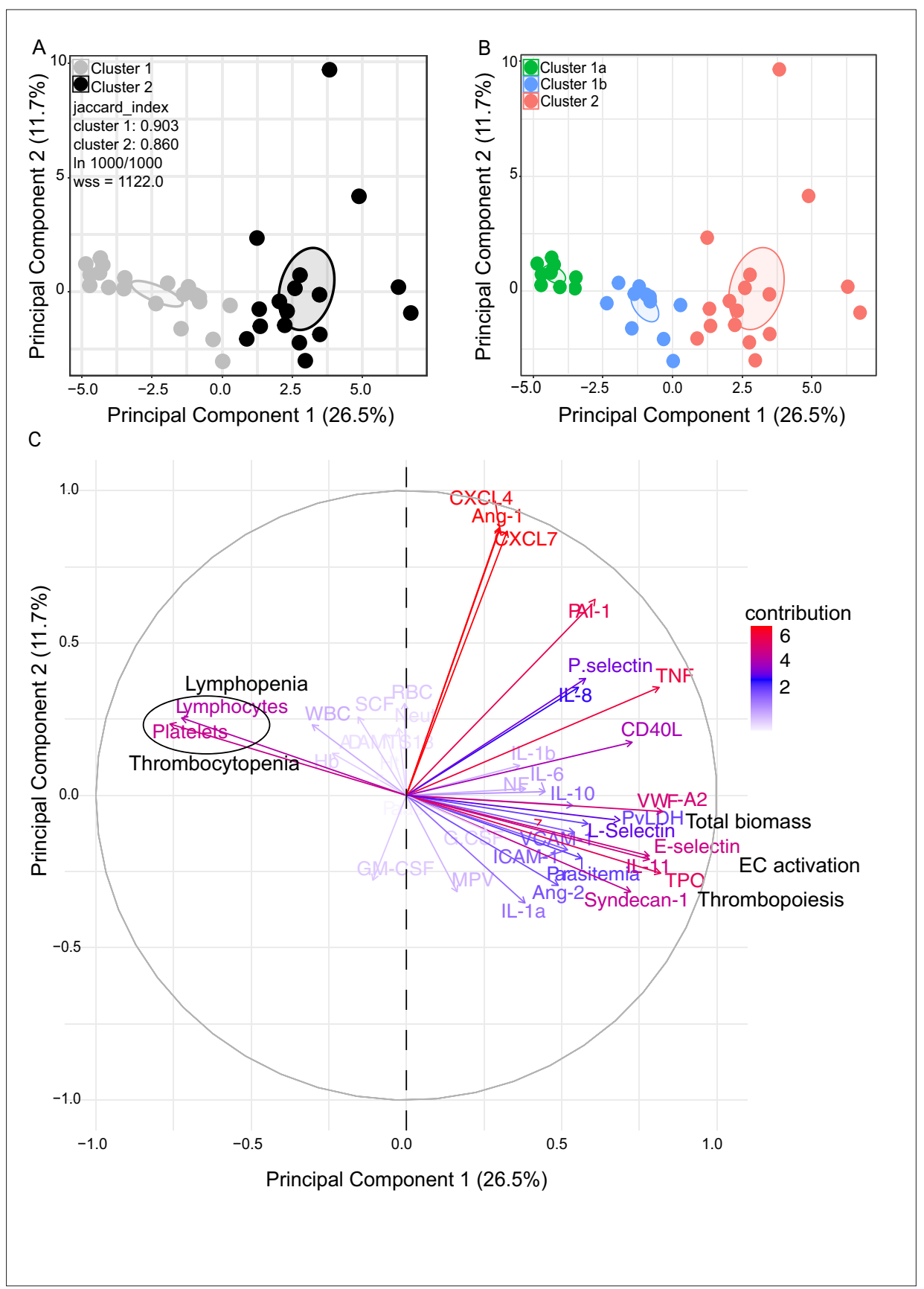

Figure 2. Characterization of heterogeneity in symptomatic $P$. vivax patients defines clusters of individuals. (A, B) Clustering of patients and healthy controls. After z-score normalization, principal component analysis (PCA) was performed for data dimensionality reduction. K-means clustering using $\mathrm{k}=2$ followed by bootstrapping (1000 times) in a PCA plot was performed and produced the most stable clusters regardless of the starting point (In 1000/1000): cluster $1=23$ individuals comprising 9 healthy donors and 14 P. vivax patients and cluster 2 comprising 17 P. vivax patients. The jaccard_index measures cluster similarity across bootstrap samples (jaccard_index ranges from 0 to 1 , an index $<0.6$ hints at a weak, unreliable cluster while $>0.85$ means generally reliable). As indicated in the PCA plot, $k=2$ gives stable clusters for all configurations (jaccard_index 0.9 and 0.86) and withinss (wss) $=1122$. Open ovals represent $95 \%$ confidence interval ellipses around group mean points. PCA was performed for data dimensionality reduction, in parallel with K-means clustering ( $\mathrm{k}$ ) followed by bootstrapping (1000 times). Open ovals represent $95 \%$ confidence interval ellipses around group mean points. (B) The resulting clusters represent healthy controls (1a) and patients (1b, 2). (C) Contribution of variables to clustering. In the circular plot, the correlation between each input variable and principal components is used as coordinates (loading score). Plots show how covariables determine patient distribution in the PCA plot.

Figure 2 continued on next page 
Figure 2 continued

The online version of this article includes the following figure supplement(s) for figure 2:

Source data 1. Correlation (loading score) of variables to principal components.

Figure supplement 1. Principal component analysis metrics.

Figure supplement 2. Methods determining the number of clusters best representing the data.

Figure supplement 2-source data 1. Measurements of $\mathrm{K}$-means cluster stability, using $\mathrm{k}=2, \mathrm{k}=3$, and $\mathrm{k}=4$ clusters, via bootstrapping.

Vivax ${ }^{\text {low }}$ (representing patients with low P. vivax biomass), and cluster two as Vivax ${ }^{\text {high }}$ (representing patients with high $P$. vivax biomass).

\section{Different levels of haematological alterations between Vivax ${ }^{\text {high }}$ and Vivax ${ }^{\text {low }}$ patients}

The three clusters were not significantly different in patient age (median: 33; IQ 25-75: 22-57), gender ( $80 \%$ male; $20 \%$ female in each cluster), average days of symptoms when samples were

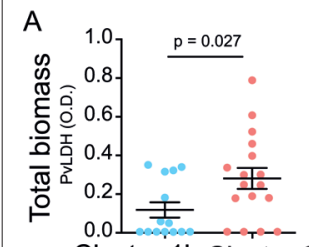

Cluster $1 \mathrm{~b}$ Cluster 2

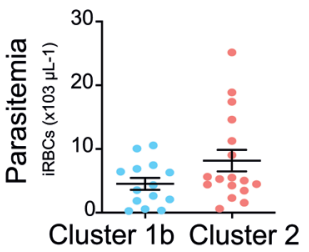

D

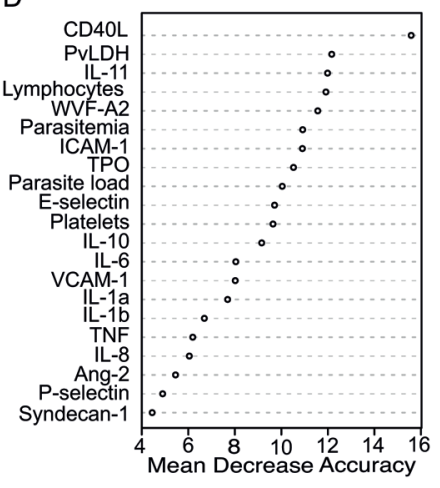

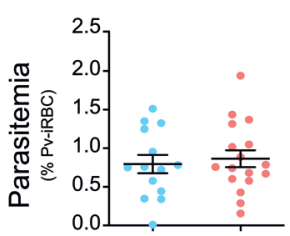

Cluster $1 \mathrm{~b}$ Cluster 2

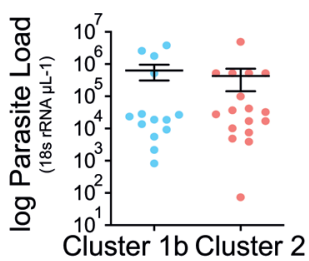

$\mathrm{E}$

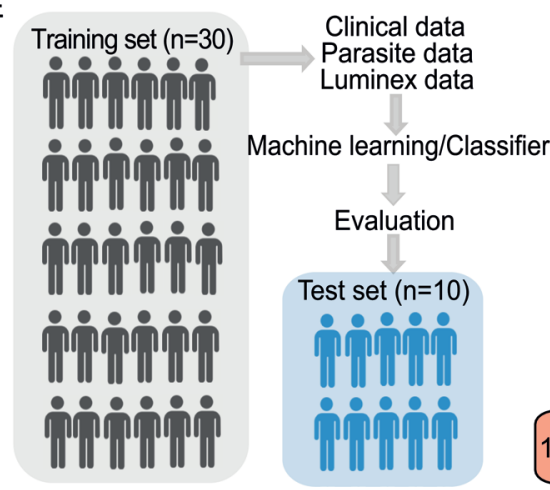

Total parasite biomass Peripheral parasitemia

Total parasite biomass Parasite load

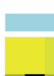
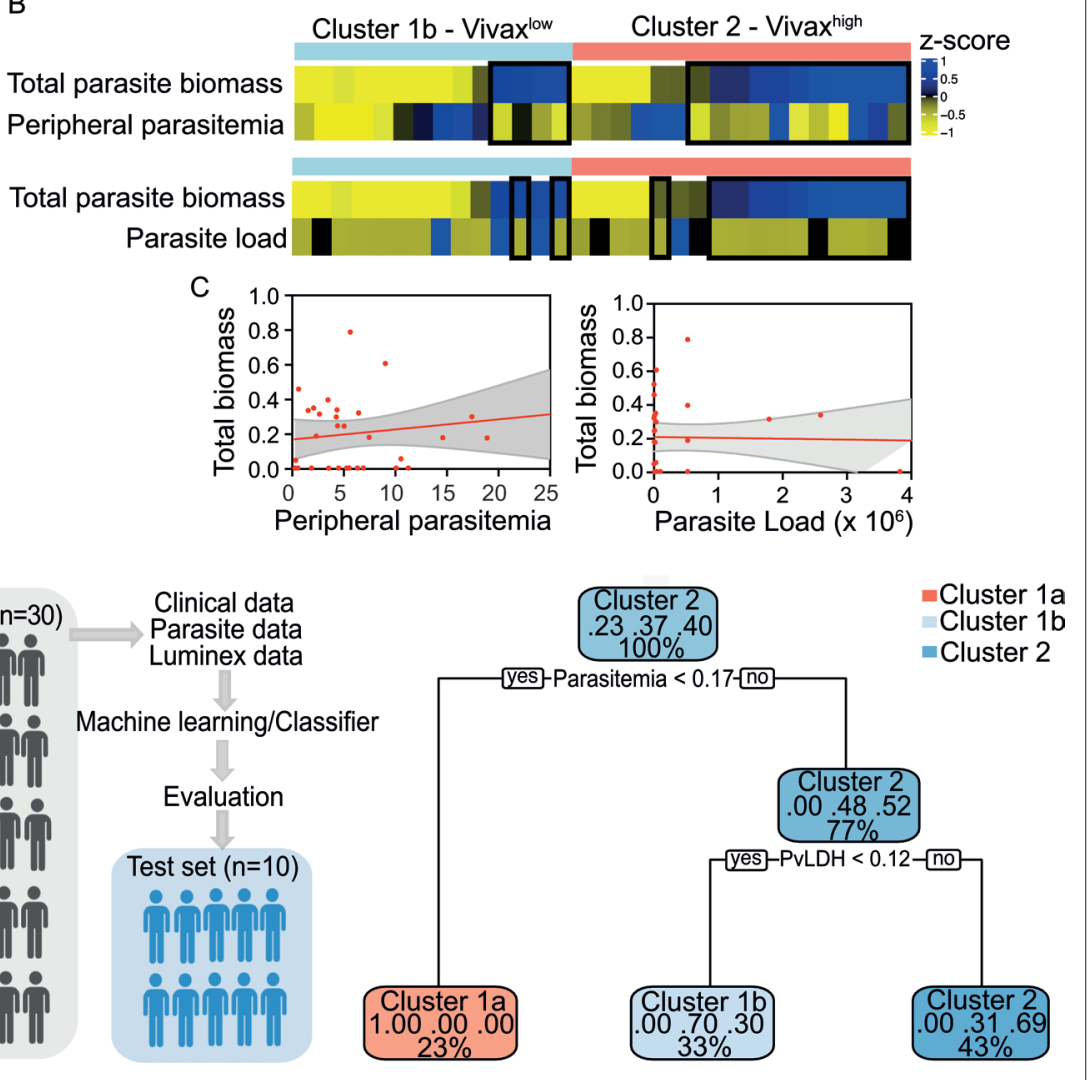

Figure 3. Unsupervised clustering analysis reveals two $P$. vivax patient subgroups that differ in parasite biomass. (A) Parasite parameters vs. patient clusters. Comparison of the two patient clusters (clusters $1 \mathrm{~b}$ and 2 ) across parasite parameters reveals significant differences with total parasite biomass (P. vivax lactate dehydrogenase [PvLDH]) but not peripheral parasitaemia or parasite load (copies of $18 S \mathrm{rRNA} / \mu \mathrm{L}$ of blood). (B) Parasite biomass vs. parasitaemia across clusters. Heatmap represents z-scores of PvLDH with peripheral parasitaemia or parasite load, respectively. Black boxes highlight patients with relatively lower peripheral parasitaemia compared to PVLDH levels, indicating the underestimation of total parasite biomass based on peripheral parasitaemia values. (C) Correlation between parasite biomass and parasitaemia. Scatter plot showing lack of correlation between PvLDH and peripheral parasitaemia or parasite load, respectively. Regression line in red, with 95\% confidence interval shown in shaded grey. (D, E) Predicting parasite clusters. (D) Top parameters prioritized by random forest analysis ranked by the mean decrease in accuracy. (E) Best-fit decision trees and random forest machine learning models corroborate PVLDH value as the most important parasite signature in segregating patients into clusters $1 \mathrm{~b}$ and 2. Cut-off values of the attribute that best divided groups were placed in the root of the tree according to the parameter value. The total of classified registers for each class and the percentage of observations used at that node are given in each terminal node. 


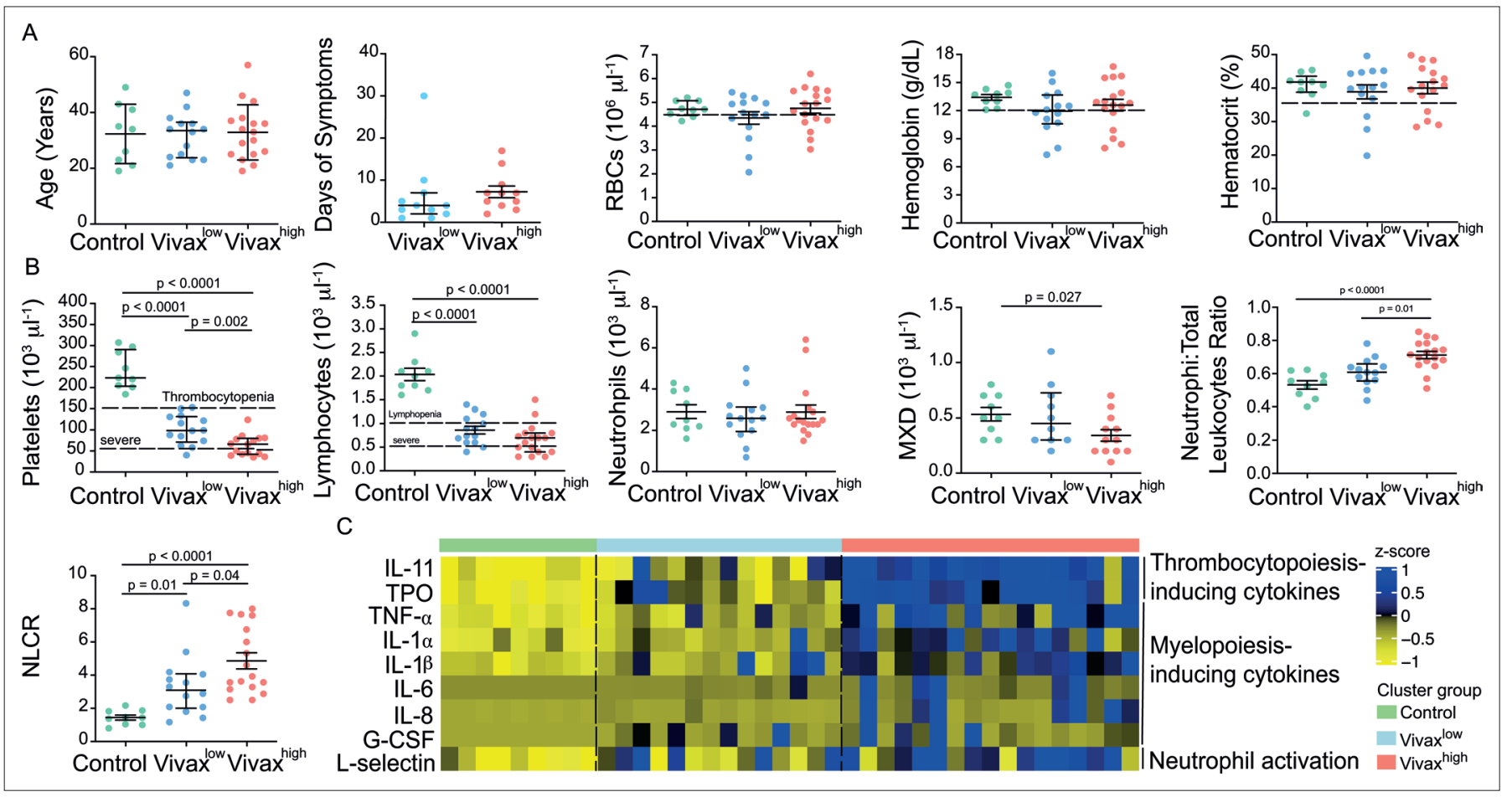

Figure 4. More severe haematological alterations in Vivax ${ }^{\text {high }}$ compared to Vivax ${ }^{\text {low }}$ patients. (A) Patient data and haematological parameters. Comparison of patient age, average days of symptoms when samples were collected, haemoglobin levels, haematocrit, or RBC counts across patient clusters (Control: $n=9$; Vivax ${ }^{\text {low }}: n=14$; Vivax high $: n=17$ ). Data are depicted as plots showing individual values and the median (black lines) and the interquartile range. (B) Blood cell counts. Comparison of differential haematological counts across clusters. Shown are numbers of platelets, lymphocytes, neutrophils, and monocytes, basophils, and eosinophils (MXD), neutrophil to total leukocyte ratio, and neutrophil to lymphocyte ratio (NLCR). Top dashed lines mark the minimal threshold for normal reference values, while bottom dashed lines mark the threshold for severe lymphopenia and thrombocytopenia, respectively. Parameters are depicted as plots showing individual values and the median (black lines) and the interquartile range. One-way analysis of variance with Bonferroni-corrected multiple comparisons test was performed. p-Value is indicated above the graph when reached significance of $p<0.05$. (C) Cytokine response and neutrophil activation across clusters. Heatmap represents z-scores obtained by centering values of Luminex data. Shown are thrombopoiesis-inducing cytokines, myelopoiesis-inducing cytokines, and neutrophil activation markers. Biomarker concentrations were normalized (scale function in R), and the average scaled value is showed in blue and yellow scales. Blue shading represents the highest average scaled value, and yellow shading represents the lowest average scaled value. Each column (i.e. individual) in the heatmap is matched with colour-coded cluster assignment: Cluster Control - green bar; Cluster Vivax ${ }^{\text {low }}$ - blue bar; and Cluster Vivax ${ }^{\text {high }}$ - red bar.

The online version of this article includes the following figure supplement(s) for figure 4:

Figure supplement 1. Increase of thrombopoiesis- and myelopoiesis-inducing cytokines in the plasma of Vivax high patients.

collected, haemoglobin levels, haematocrit, or RBC counts, indicating that these parameters are not confounders accounting for the differences observed between the clusters (Figure 4A). However, systematic analysis of haematological parameters between Vivax ${ }^{\text {high }}$ and Vivax ${ }^{\text {low }}$ patients revealed significant differences. Vivax ${ }^{\text {high }}$ patients showed a more intense reduction in platelet counts when compared to Vivax ${ }^{\text {low }}$ patients (Vivax ${ }^{\text {high }} 63,000 \pm 6413$ vs. Vivax ${ }^{\text {low: }} 100,700 \pm 9381 ; p=0.002$ ), with a higher frequency of patients with severe thrombocytopenia (Vivax ${ }^{\text {high }} 47 \%$ vs. Vivax ${ }^{\text {low }} 8 \%$ ) (Figure 4B). Although not significant, there was a trend in the reduction of lymphocyte counts in Vivax ${ }^{\text {high }}$ patients when compared to Vivax ${ }^{\text {low }}$, with $88 \%$ of Vivax ${ }^{\text {high }}$ patients presenting lymphopenia versus $64 \%$ in Vivax ${ }^{\text {low }}$ patients. In addition, we observed a fourfold increase in the frequency of patients with severe lymphopenia in the Vivaxigh cluster compared to Vivax ${ }^{\text {low }}$ patients (Figure 4B). While there was no change in the number of circulating neutrophils in the different clusters of individuals, mixed cell counts (MXD), a parameter representing monocytes, basophils, and eosinophils numbers, were significantly reduced in Vivax ${ }^{\text {high }}$ patients. As a result, there was a significant enrichment of neutrophils in the leukocyte fraction in the blood of Vivax ${ }^{\text {high }}$ patients as well as a higher NLCR (Figure 4B).

In parallel to more severe thrombocytopenia in Vivaxhigh patients, plasma levels of cytokines inducing megakaryocytic differentiation in the BM, thrombopoietin (TPO), and IL-11 were significantly 
increased in this cluster (Figure 4C, Figure 4-figure supplement 1). In accordance with the pattern of immune cell fractions in the peripheral blood of $P$. vivax patients, the Vivax ${ }^{\text {high }}$ cluster showed a significant increase in the levels of proinflammatory cytokines associated with induction of myeloid-biased haematopoietic stem cell (HSC) differentiation and inhibition of lymphopoiesis in BM (e.g. TNF- $\alpha$, IL-1 $\alpha$, IL-1 $\beta$, IL-6, IL-8; Figure 4C, Figure 4-figure supplement 1; Boiko and Borghesi, 2012; Chiba et al., 2018; Kovtonyuk et al., 2016). In addition, Vivax ${ }^{\text {high }}$ patients had increased circulating levels of G-CSF, a major mediator of HSC-biased myelopoiesis and the neutrophil activation marker, L-selectin (Figure 4C, Figure 4-figure supplement 1; Soehnlein et al., 2017; Ivetic, 2018; Crockett-Torabi et al., 1995). Together, these Luminex data support the haematological measurements, suggesting that a compensatory response is mounted in the BM to counterbalance the massive decrease of platelets in periphery. Upregulation of cytokines inducing myelopoiesis, while inhibiting lymphopoiesis (Boiko and Borghesi, 2012; Chiba et al., 2018; Kovtonyuk et al., 2016), might also explain the decrease of lymphocyte counts and enrichment of activated neutrophils in the peripheral circulation of $P$. vivax patients.

\section{Elevated circulating markers of EC activation and damage in Vivax ${ }^{\text {high }}$ compared to Vivax ${ }^{\text {low }}$ patients}

Patient clustering indicated that Vivax ${ }^{\text {high }}$ patients have increased levels of EC markers in the plasma compared to Vivax ${ }^{\text {low }}$ patients (Figure 2C). Previous studies indicate that EC activation and damage might contribute to thrombocytopenia and inducing haematopoiesis, resulting in HSC differentiation directed towards myelopoiesis (Lacerda et al., 2011; de Mast et al., 2009; de Mast et al., 2007; Boiko and Borghesi, 2012; Chiba et al., 2018; Kovtonyuk et al., 2016; Graham et al., 2016; DosSantos et al., 2020; Lazzari and Butler, 2018). In our cohort, circulating levels of EC adhesion molecules (ICAM-1, VCAM-1, E-selectin, and P-selectin) and other EC activation markers and procoagulant molecules (Ang-2, VWF-A2, CD40L, and PAI-1) were significantly increased in the plasma of Vivax ${ }^{\text {high }}$ patients compared to Vivax ${ }^{\text {low }}$ patients and healthy controls (Figure 5A, Figure 5-figure supplement $1 \mathrm{~A}$ and B). Likewise, Syndecan-1, a marker of EC glycocalyx breakdown (i.e. damage of EC plasma membrane; Yeo et al., 2019; Pillinger and Kam, 2017), was significantly increased in Vivax ${ }^{\text {high }}$ but not in Vivax ${ }^{\text {low }}$ patients (Figure 5A, Figure 5-figure supplement $1 \mathrm{C}$ ).

To independently test whether host factors and/or parasite products present in the plasma of the different patient groups can directly induce changes in ECs, we stimulated primary human umbilical vein endothelial cells (HUVECs) with pools of plasma from either Vivax ${ }^{\text {high }}$ patients, Vivax ${ }^{\text {low }}$ patients, or healthy controls. These experiments demonstrated that only pooled plasma from Vivax ${ }^{\text {high }}$ patients induces significant transcriptional upregulation of EC activation markers ICAM-1, IL-1 $\alpha$, and IL-8 along with downregulation of Ang-1, ADAMTS13, and NOS3 (eNOS) in HUVECs (Figure 5B, Figure 5figure supplement 1D). In contrast, expression of Syndecan-1 and VEGF, two indicators of vascular damage, was not affected by either treatment (Figure 5B, Figure 5-figure supplement 1D). Similarly, electric cell-substrate impedance sensing (ECIS) assays did not detect differences in functional perturbations in the endothelial cellular monolayer upon incubation with $P$. vivax pooled plasma when compared to control pooled plasma (Figure 5C). In contrast, flow cytometry and immunofluorescence assays performed with stimulated HUVECs revealed increased prevalence and protein expression levels of EC activation markers ICAM-1 and VCAM-1 upon exposure with Vivaxigh pooled plasma (Figure 5D, Figure 5-figure supplement 1E), in support of qRT-PCR data. These data indicate that local EC activation, mediated by direct or indirect interactions with parasitized RBCs, can be measured systemically.

\section{Indirect evidence for parasite-induced changes in deep tissues}

To further investigate the interplay between host biomarkers and associated cellular responses as well as parasite parameters, we constructed a network of interactions based on Pearson's correlations with absolute correlation coefficient above 0.5 and p-value $<0.05$ (Figure 6A). In addition, we also performed hierarchical clustering on matrices of Pearson's correlations ( $p$-value $<0.01$ ) with selected modules of parasite and host parameters (Figure 6B). Data from Vivax ${ }^{\text {low }}$ and Vivax ${ }^{\text {high }}$ patient subgroups were combined for this analysis as they similarly contribute to the associations we found so far (Figure 6-figure supplement 1). 


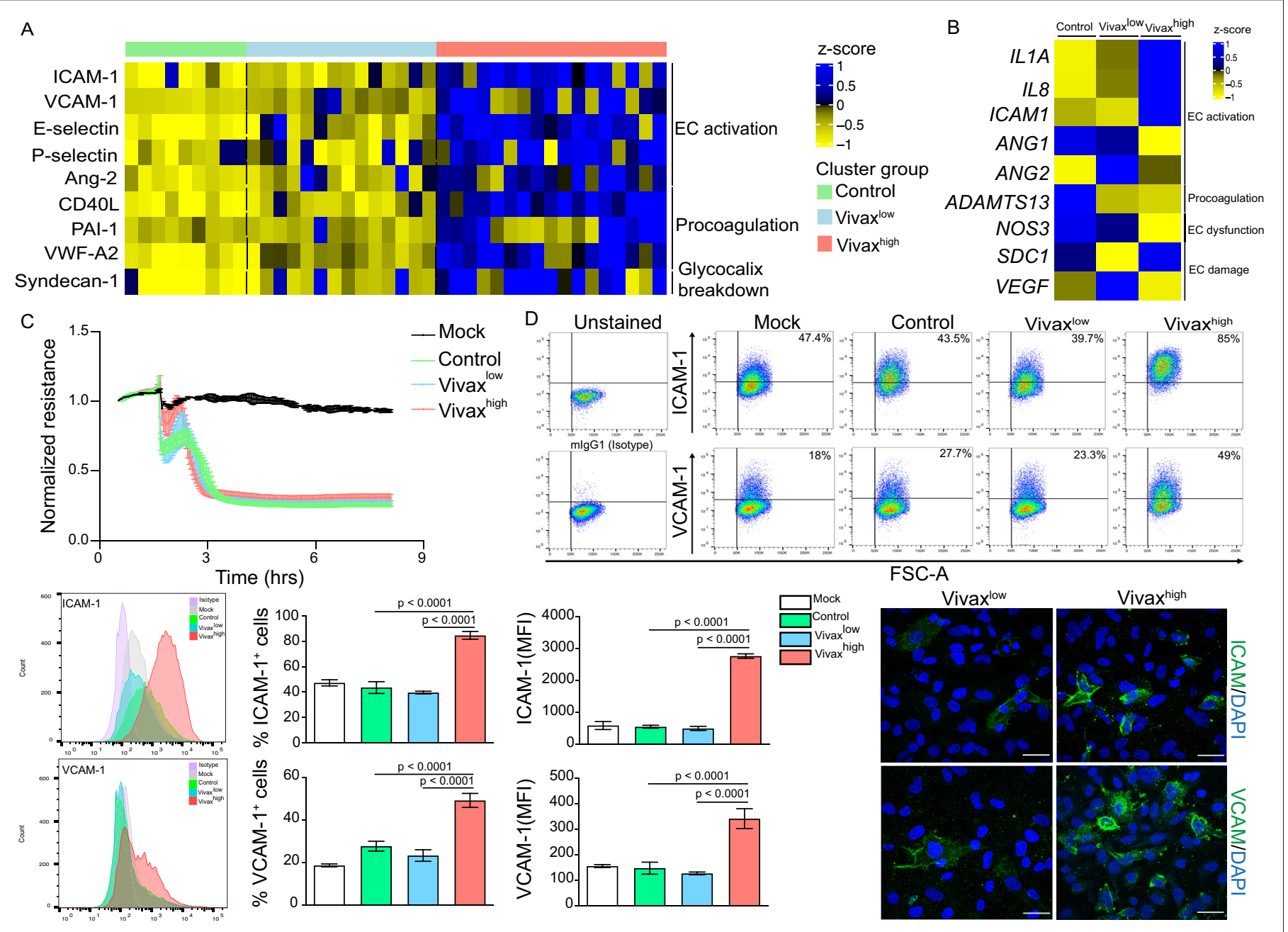

Figure 5. Elevated circulating markers of endothelial cell (EC) activation and damage in Vivax ${ }^{\text {high }}$ compared to Vivax ${ }^{\text {low }}$ patients. (A) Endothelial changes across clusters: Luminex. Heatmap represents z-scores obtained by centering values of Luminex data. Shown are markers of EC activation, procoagulation, and glycocalyx damage. Each column (each individual) in the heatmap is matched with colour-coded cluster assignment: Cluster Control - green bar; Cluster Vivax ${ }^{\text {low }}$ - blue bar; and Cluster Vivax high - red bar. (B) Endothelial changes across clusters: qRT-PCR. Transcriptional response of human umbilical vein endothelial cells (HUVECs) incubated for $6 \mathrm{hr}$ with $30 \% \mathrm{v} / \mathrm{v}$ pooled plasma from different clusters. Heatmap reflects relative mRNA expression intensity (average scaled value) after results were normalized to GAPDH housekeeping gene expression and untreated condition (mock). Data shown represent the mean of three independent experiments. For each experiment, two technical replicates were performed for each condition. (C) Endothelial changes across clusters: impedance changes. Endothelial monolayer integrity was measured during $20 \% \mathrm{v} / \mathrm{v}$ of pooled plasma incubation. Each line represents the mean \pm SD of normalized resistance of HUVECs measured by electric cell-substrate impedance sensing $(E C I S)$ at $4000 \mathrm{~Hz}$. Data shown are representative of three independent experiments. For each experiment, two technical replicates were performed for each condition. (D) Endothelial changes across clusters: imaging and flow cytometry. HUVECs were incubated for $18 \mathrm{hr}$ with $30 \% \mathrm{v} / \mathrm{v}$ of pooled plasma of individuals in the different clusters or left untreated (mock). Percentage of cells expressing EC activation markers (adhesion molecules) ICAM and VCAM as well as quantification of protein expression was determined by flow cytometry and immunofluorescence analysis (scale bar $=33 \mu \mathrm{M}$ ). Isotype antibodies were used as control to define positive populations. Significance was calculated for comparisons between conditions at the corresponding time point. One-way analysis of variance statistical test with Tukey's corrected multiple comparisons test was performed. p-Value is indicated above the graph when $p<0.05$. Data shown are representative mean \pm SEM of three independent experiments.

The online version of this article includes the following figure supplement(s) for figure 5 :

Figure supplement 1. Increase of markers of endothelial cell (EC) activation, damage (glycocalyx breakdown), and procoagulation in the plasma of Vivax ${ }^{\text {high }}$ patients.

Figure supplement 2. Haemolysis potentiates Vivax ${ }^{\text {high }}$-induced endothelial cell (EC) activation. 


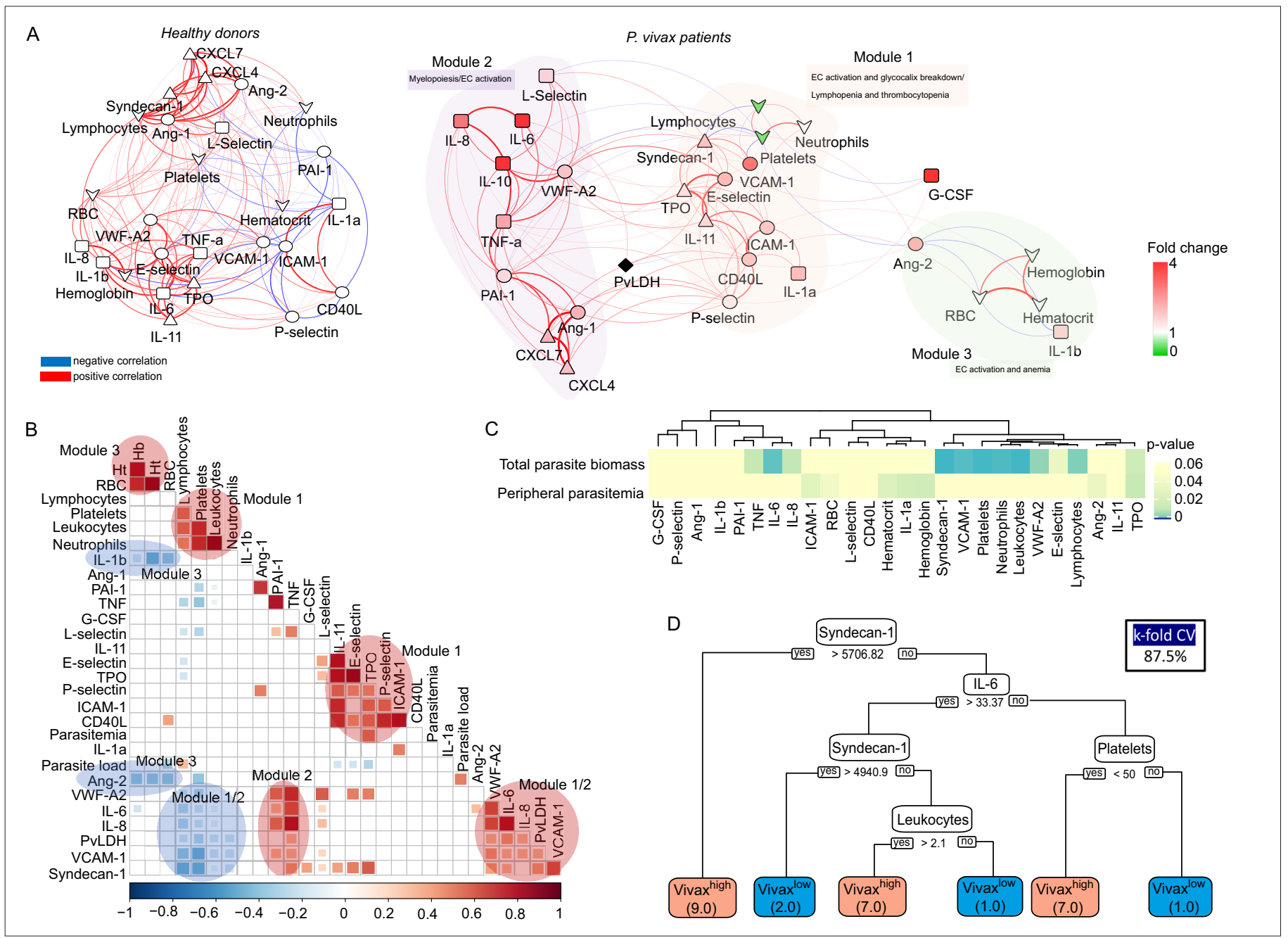

Figure 6. Network analysis and clustering of parasite and host signatures indicate parasite-induced changes in deep tissues. (A) Network analysis. Networks of the Pearson's correlations (absolute coefficient above 0.5 and p-value $<0.05$ ) between parasite biomass $(P$. vivax lactate dehydrogenase $[\mathrm{PvLDH}]$ ) and host signatures in healthy donors (left graph) and in P. vivax-infected patients (right graph), using a force-directed layout. The symbols of the nodes represent biological functions: triangle represents markers of platelet activation and thrombopoiesis-inducing cytokines; $V$ shape represents haematological parameters (neutrophil, lymphocyte, and platelet counts); circles represent endothelial cell activation markers; squares represent myelopoiesis-inducing cytokines and neutrophil activation markers. The colours in the nodes represent the fold change in relation to control levels. Because healthy donors do not have parasitaemia, PvLDH node is represented in black. Each connecting line (edge) represents a significant interaction detected by the network analysis using R. Correlation strength is represented by edge colour transparency and width. Positive correlations are represented by red edges, and negatives correlations are represented by blue edges. (B, C) Correlation matrix and heatmap. (B) Representative image of Pearson's correlation matrix calculated for all P. vivax patients. Only correlations with $p$-value $<0.01$ are shown, and hierarchical clustering was applied. Red circles highlight positive correlations in the functional modules depicted in (A), and blue circles highlight negative correlations in the functional modules also depicted in (A). (C) Heatmap showing p-values of the correlations between different parasite parameters, parasite biomass $(\mathrm{PVLDH})$, and peripheral parasitaemia and host signatures (haematological and Luminex parameters). (D) Decision tree model. Best-fit classification tree model generated with the C4.5 algorithm showing Syndecan-1, IL-6, and platelet counts are the dominant variables capable of predicting total parasite biomass in P. vivax patients. Cut-off values of the attribute that best divided groups were placed in the root of the tree according to the parameter value $(\mathrm{pg} / \mathrm{mL}$ for soluble markers or number of cells $\times 1000 / \mu \mathrm{L}$ of blood for platelet counts). The total of classified registers for each class is given in parentheses for each terminal node with the k-fold cross-validation ( $k$-fold CV) accuracy indicated.

The online version of this article includes the following figure supplement(s) for figure 6:

Figure supplement 1. Representative images of Pearson's correlation matrix calculated separately for each P. vivax patient cluster.

Figure supplement 2. Validation of patients' clusters and correlations when segregating patients based on thrombocytopenia severity.

Figure supplement 3. Validation of patients' clusters and correlations when segregating patients based on lymphopenia severity. 
Similar to a previous study with P. vivax patients and healthy donors from an endemic area in Brazil (Mendonça et al., 2013), our analysis revealed a dense network of interactions with homogenous and centralized topology among the biomarkers in healthy donors (Figure 6A, Supplementary file 1). The network topology is drastically altered in symptomatic $P$. vivax patients, largely due to the introduction of parasite parameters in the patient graph (Figure 6A, Supplementary file 1). The network analysis revealed a decentralized topology, lower complexity and connectivity between the edges with data from $P$. vivax patients compared to the highly dense, homogenous and centralized network graph of healthy donors (91 edges vs. 166 edges, respectively). Of note, the network pattern described in our study is similar to protein-protein-associated networks described previously in $P$. vivax malaria and in other clinical contexts (Mendonça et al., 2013; Frankenstein et al., 2006). Interestingly, due to its decentralized and heterogenous patterns of interactions, the network graph of $P$. vivax patients is separated into three modules of strong interactions, with closely related biological functions. Module 1 is formed by markers of EC activation and damage, together with lymphocyte, platelet, and neutrophil counts in addition to the megakaryocyte differentiation-inducing cytokines (TPO and IL-11) (Figure 6A). In support of the role of EC activation and damage in the haematological changes observed in this cohort, hierarchical clustering revealed a positive correlation between adhesion molecules VCAM-1 and E-selectin and EC glycocalyx breakdown (Syndecan-1) (Figure 6B). In addition, VCAM-1, E-selectin, Ang-2 and VWF-A2, and Syndecan-1 are negatively correlated with platelet and lymphocyte counts, while ICAM-1 is positively correlated with neutrophil counts (Figure 6A and B). Module 2 is formed by proinflammatory cytokines with myelopoiesis-inducing effects and molecules associated with platelet activation and coagulation cascades (Figure 6A and B). Interestingly, EC activation markers and Syndecan-1 (EC damage) from module 1 also display positive correlations with myelopoiesis-inducing cytokines from module 2 (Figure 6B). Finally, module 3 is formed by Ang-2 and the proinflammatory cytokine IL-1 $\beta$ negatively associated with haemoglobin, haematocrit, and RBC numbers (anaemia markers) (Figure 6A and B). Most notably, PvLDH connects the two main functional modules 1 and 2 (Figure 6A and B). Accordingly with Figures 2, 6A and B, the biological significance of total parasite biomass, but not peripheral parasitaemia or parasite load, in affecting host response is also corroborated by the high significant and positive associations of PvLDH with multiple host parameters, including Syndecan-1 (EC damage), VCAM-1, VWF (EC activation and platelet pooling), and IL-6, IL-8, and TNF- $\alpha$ (inflammation and myelopoiesis-inducing cytokines) (Figure 6C). Meanwhile, platelet, lymphocyte, and neutrophil counts are negatively correlated with high significance ( $p$-value $<0.0001$ ) with total parasite biomass, but not with peripheral parasitaemia or parasite load (Figure 6C). The association between endothelial activation, Syndecan-1, and parasite biomass (PvLDH) indicates a positive feedback loop between glycocalyx breakdown, activation of endothelial receptors such as ICAM-1 and VCAM-1, and parasite accumulation in deep tissues (Carvalho et al., 2010; Lopes et al., 2014). Similar to Figure 2E, application of a best-fit classification tree model demonstrated that Syndecan-1, IL-6, and platelet counts are the most dominant predictor attributes capable of classifying $P$. vivax patients based on total parasite biomass levels (Figure 6D). Using this model, all P. vivax patients were correctly classified into either low (Vivax ${ }^{\text {low }}$ ) or high (Vivax ${ }^{\text {high }}$ ) total parasite biomass (PvLDH). In turn, PvLDH is a relevant predictor attribute (high information gain) in predicting thrombocytopenia severity, and it is associated with increased severity of thrombocytopenia and lymphopenia in our cohort (Figure 6-figure supplement 2, Figure 6-figure supplement 3). Together, these data further support the hypothesis that a parasite population outside of circulation, as represented by total parasite biomass, is driving the host response including EC activation and damage as well as haematological disturbances (i.e. lymphopenia, thrombocytopenia, and anaemia) in P. vivax patients (Figure 6-figure supplement 2, Figure 6-figure supplement 3).

\section{Discussion}

In this study, we performed a comprehensive analysis of host and parasite signatures detected in the plasma of a cross-sectional cohort of uncomplicated $P$. vivax malaria. Initial analysis of a series of circulating host biomarkers revealed significant levels of thrombocytopenia, lymphopenia, and anaemia, as well as EC activation and damage across $P$. vivax patients compared to healthy controls. Deconvolution of heterogeneity across patients revealed two patient subgroups (Vivax ${ }^{\text {high }}$ and Vivax ${ }^{\text {low }}$ ) characterized by differences in total parasite biomass (based on circulating PvLDH levels) but not peripheral parasitaemia (based on blood smears). We observed a significant correlation between total 
parasite biomass (but not peripheral parasitaemia) and systemic levels of markers of EC activation and damage and haematopoietic perturbations. In addition, by applying a supervised machine learning tree-structured model, we were able to associate EC damage and thrombocytopenia with parasite biomass. In agreement with a previous study (Barber et al., 2015; Silva-Filho et al., 2021), our observations further suggest that total parasite biomass as measured by PvLDH is a better predictor of $P$. vivax host responses and pathogenesis than peripheral parasitaemia. Furthermore, these findings support the emerging paradigm of a major $P$. vivax parasite reservoir outside of circulation, particularly in the haematopoietic niche of BM and spleen (Silva-Filho et al., 2021).

The existence of a significant $P$. vivax reservoir outside of circulation was first predicted by disproportionately high PvLDH levels in peripheral circulation compared to parasitaemia by blood smear (particularly in patients with complicated outcomes) and by modelling using experimental Plasmodium cynomolgy infections in NHPs (Barber et al., 2015; Fonseca et al., 2017). Recent studies provide direct evidence that BM and spleen represent the major reservoir of parasite biomass in $P$. vivax infection (Obaldia et al., 2018; Baro et al., 2017; Brito et al., 2020; Kho et al., 2021a; Kho et al., 2021b). PvLDH is produced by viable or recently killed parasites and hence considered a proxy for ongoing rather than past infection (Barber et al., 2015; Druilhe et al., 2007). PvLDH antigen capture ELISA established a direct relationship between pLDH levels and $P$. vivax parasitaemia in ex vivo experiments, demonstrating that pLDH reflects total $P$. vivax parasite biomass (Druilhe et al., 2007). Our study further explores the relevance of PvLDH as a prognostic marker of host perturbations and disease severity, with a particular focus on markers of changes in the haematopoietic niches of $\mathrm{BM}$ and spleen. A major observation in the network graph of $P$. vivax patients is the central position of the total parasite biomass marker PvLDH due to its equally strong interactions with the two main functional modules 1 and 2. Given that the haematopoietic niches of the BM and the spleen are the major reservoir of parasite biomass, interactions of PVLDH with these two main modules indicate an interplay between parasite infection in these niches and endothelial activation/damage as well as the proinflammatory response that results in myeloid-biased differentiation, thrombocytopenia, and lymphopenia. Furthermore, the highly significant and positive associations between endothelial activation, Syndecan-1, and parasite biomass (PvLDH) indicate a positive feedback loop between glycocalyx breakdown, activation of endothelial receptors such as ICAM-1 and VCAM-1, and parasite accumulation in deep tissues. Vivax ${ }^{\text {High }}$ patients show higher plasma levels of all these markers. Consistent with previous reports (Yeo et al., 2019; Barber et al., 2021), we propose that elevated EC activation and glycocalyx damage increases the exposure of adhesion molecules, which in turn favours endothelial cytoadherence of $P$. vivax-infected RBCs, particularly in the splenic red pulp cords and in the BM (Kho et al., 2021a; Introini et al., 2018; Hempel et al., 2017; Toda et al., 2020). Accordingly, application of a best-fit classification tree model identifies Syndecan-1 as a putative host biomarker (EC glycocalyx breakdown marker) predicting total parasite biomass in $P$. vivax patients. We hypothesize that elevated endothelial activation and damage in Vivax ${ }^{\text {High }}$ patients results in increased cytoadherence of $P$. vivax iRBCs and hence accumulation and growth in deep tissues, thus reducing the fraction of the parasite biomass in circulation.

In contrast to $P$. falciparum-infected individuals, a wide range of complicated clinical syndromes occur in P. vivax patients even at low or subpatent parasitaemia (Baird, 2013), thus indicating that peripheral parasitaemia is a poor predictor of clinical outcomes. Two lines of evidence support our conclusion that severity of infection is dependent on parasite biomass instead. First, the discrepancy between PVLDH levels and peripheral parasitaemia determined by blood smears is more evident in $P$. vivax-infected patients with complicated outcomes: the ratio of plasma pLDH to peripheral parasitaemia is sixfold higher than in non-severe patients. The same comparison between severe and non-severe P. falciparum patients reveals only a 1.4-fold difference (Barber et al., 2015). Second, although thrombocytopenia and lymphopenia are not included in the World Health Organization (WHO) criteria for defining severe malaria, it has been associated with severe manifestations and the need for blood and platelet transfusions in severe vivax malaria. This points out their clinical relevance in malaria diagnosis and treatment (Lacerda et al., 2011; Naing and Whittaker, 2018; Gerardin et al., 2002; Kochar et al., 2010; Kochar et al., 2005), suggesting that these haematological complications could be explored as markers of severity for this species. Both severe thrombocytopenia and lymphopenia were more frequent in patients in cluster 2 (Vivax ${ }^{\text {high}}$ ) in our study. By integrating these clinical perturbations with host biomarker measurements and parasite parameters, we demonstrated 
the high attribute value of total parasite biomass in predicting the severity of thrombocytopenia and lymphopenia and highly significant correlations with endothelial activation, glycocalyx breakdown, and other markers of inflammation.

Thrombocytopenia, lymphopenia, and anaemia are the most frequent $P$. vivax- and $P$. falciparumassociated haematological complications (Lacerda et al., 2011; Naing and Whittaker, 2018; Rodriguez-Morales et al., 2005; Tangpukdee et al., 2008). In our cohort, 34, 85, and 87\% of patients exhibited anaemia, lymphopenia, and thrombocytopenia, respectively. Various mechanisms have been proposed to explain the damage or excessive removal of platelets in $P$. vivax infection, including oxidative stress, platelet phagocytosis, IgG binding to platelet-bound malaria antigens, spleen pooling, or increased circulating nucleic acids levels (Lacerda et al., 2011; Naing and Whittaker, 2018; Kochar et al., 2010; Andrade et al., 2010). EC activation and damage also plays a role in intravascular platelet agglutination and increased platelet clearance from the circulation (Park et al., 2003; Punnath et al., 2019). Our data also demonstrate that thrombocytopenia is associated with an increase in IL-1, IL-6, IL-8, IL-10, and TNF- $\alpha$. We also observed elevated levels of cytokines inducing megakaryocyte differentiation, TPO, and IL-11, suggesting that a compensatory response is mounted in the BM to counterbalance the massive decrease of platelets in the periphery. In contrast, the relatively large drop in peripheral lymphocyte numbers we observed in the $P$. vivax patients is likely non-specific effect, for example, pooling in the enlarged spleen rather than a response by Plasmodium-specific lymphocytes (Hviid and Kemp, 2000). Corroborating the potential role of total parasite biomass, rather than peripheral parasitaemia, in haematological disturbances (i.e. lymphopenia, thrombocytopenia, and anaemia), Figures S7 and S8 show that total parasite biomass increases accordingly with thrombocytopenia and lymphopenia severity. Patients with severe thrombocytopenia also show more severe leukopenia, lymphopenia, and mega platelets (higher MPV). In addition, plasma levels of cytokines - such as TNF- $\alpha$, IL1- $\beta$, IL-8, IL-10; EC activation/damage markers, VCAM-1, E-selectin, VWF-A2, Ang-2, Ang-2:Ang1 ratio; Syndecan-1; thrombopoiesis-inducing cytokines, TPO and IL-11; platelet activation marker, CD40L; and neutrophil activation marker, L-selectin - follow the increase in thrombocytopenia severity (Figure 6-figure supplement 2). A similar pattern is observed when stratifying patients based on lymphopenia severity (Figure 6-figure supplement 3). Interestingly, a tree-structured model demonstrated that PvLDH, along with VCAM-1 and Syndecan-1, is a relevant predictor attribute (high information gain) in predicting thrombocytopenia severity in our cohort (Figure 6-figure supplement 2).

Our data support previous studies suggesting a role for EC activation and damage in increased leukocyte adhesion, intravascular platelet agglutination with increased platelet clearance from the circulation and skewing of haematopoiesis towards the myeloid lineage (likely at the expense of lymphopoiesis) in the BM (de Mast et al., 2009; de Mast et al., 2007; Gomes et al., 2014; Boiko and Borghesi, 2012; Chiba et al., 2018; Kovtonyuk et al., 2016; Graham et al., 2016; Dos-Santos et al., 2020; Pillinger and Kam, 2017). P. vivax elicits a stronger inflammatory response and more pronounced endothelial activation when compared with other Plasmodium infections with similar or higher peripheral parasitaemia (Yeo et al., 2010); however, the role of EC activation in P. vivax pathogenesis is not yet understood. Damage of the EC plasma membrane, as represented by glycocalyx breakdown, has been associated with poor prognostic outcome in P. falciparum (Yeo et al., 2019), but there is no data available for P. vivax. In our cohort, soluble EC activation biomarkers (e.g. ICAM-1, VCAM-1, E-selectin, Ang-2, CD40L, vWF-A2) and the EC damage product, Syndecan-1, are positively correlated with thrombocytopenia, lymphopenia, anaemia, and neutrophil enrichment in the peripheral blood. In addition, these biomarkers are positively correlated with increased circulating levels of cytokines inducing megakaryocyte differentiation (e.g. IL-11 and TPO) and with cytokines inducing myeloid-biased HSC differentiation (e.g. TNF- $\alpha$, IL1- $\alpha$, IL6, IL-8, and G-CSF), suggesting both direct and indirect links between EC activation and damage and haematological perturbations. Total parasite biomass-inducing EC activation might act synergistically with inflammatory changes potentially leading to splenic platelet pooling and platelet clumping in the vasculature without DIC (Lacerda et al., 2008; Pillinger and Kam, 2017; Becker et al., 2015). Likewise, increased activation-induced cell death (AICD) in T cells, splenic T-cell accumulation (Hviid and Kemp, 2000), or decreased lymphopoiesis due to myeloid-biased HSC differentiation induced by inflammatory cytokines and EC activation in the BM (Boiko and Borghesi, 2012; Chiba et al., 2018; Silva-Filho et al., 2021) might explain the severe lymphopenia and neutrophilia in vivax patients. Together, such mechanisms could explain 
the link between parasite biomass and EC activation/damage with haematological changes observed in vivax patients that might contribute to pathogenesis and disease severity.

In a second series of experiments, we performed ex vivo stimulation of HUVECs with the plasma of the $P$. vivax cohort demonstrating that the mixture of parasite and host factors can directly induce EC activation in the absence of parasitized RBCs. Of note, functional differences between HUVECs and adult vascular endothelium, including lack of $A B O$ blood group antigen expression, have been reported (O'Donnell et al., 2000; Tan et al., 2004). Hence, EC stimulation with patient plasma may be further evaluated using primary vascular ECs.

ECs are capable of responding to pathogens by sensing pathogen-associated molecular patterns (PAMPs) through pattern-recognition receptors (PRRs), which might play a key role in inducing EC activation when detecting $P$. vivax molecules enriched in the tissues where the parasite accumulates. ECs also express specific cytokine/chemokine receptors to detect proinflammatory signals released systemically or locally by activated immune cells in response to infection (Bernardo et al., 2004; Bevilacqua, 1993). As a result, EC activation induces exocytosis of secretory granules known as Weibel-Palade bodies that leads to the release of Ang-2 and WWF, as well as transcriptional programmes that activate expression of adhesion molecules such as ICAM-1, VCAM-1, E-selectin, and secreted cytokines and chemokines (de Mast et al., 2007; Bernardo et al., 2004; Bevilacqua, 1993). However, EC pathophysiology is complex, and changes represent a heterogenous spectrum ranging from simple perturbation to activation and even EC damage (de Mast et al., 2009). Our Luminex data clearly confirm such heterogeneity in the spectrum of EC changes due to $P$. vivax infection, with systemic increase of markers of EC activation and damage only detected in Vivax ${ }^{\text {high }}$ patients. The ex vivo data show that increased systemic host proinflammatory factors and/or parasite products can alter EC properties, including activation of adhesion molecules and proinflammatory cytokines and downregulation of ADAMTS13. In contrast, vascular integrity was not affected. These data indicate that systemic inflammatory responses in $P$. vivax patients can lead to local EC activation but not vascular damage, central events in malaria pathogenesis. It is likely that other circulating factors that we have not directly measured in our study are also contributing to EC activation and vascular permeability. In particular, extracellular vesicles (EV) originating from ECs, platelets, and RBCs are present during malaria infection and are known to modulate the host immune response to the parasite (Toda et al., 2020; Mantel et al., 2016; Mantel et al., 2013). In P. falciparum, infected RBCs release EVs containing immunogenic parasite antigens, which activate macrophages, induce neutrophil migration, and alter endothelial barrier function (Mantel et al., 2016; Mantel et al., 2013). In P. vivax, plasma-derived EVs from iRBCs are taken up by human spleen fibroblasts (hSFs). This event signals NF-kB translocation and upregulation of ICAM-1 expression, facilitating cytoadherence of $P$. vivax-infected reticulocytes (Toda et al., 2020).

Although our study lacks longitudinal information, the findings might have clinical implications during and after treatment of vivax malaria. Several case reports demonstrate progressive clinical deterioration after commencement of treatment in $P$. vivax patients, associated with parasite killing that result in haemolysis of iRBCs and intravascular inflammation and oedema in response to the products released from these cells (Anstey et al., 2007; Anstey et al., 2002; Tan et al., 2008; Val et al., 2017). Patients presenting with a strong host response during acute infection might therefore be at increased risk of deteriorating and developing severe symptoms after commencement of treatment (Figure 5-figure supplement 2). Thus, identification of unique biological signatures in $P$. vivax patients might help to build rational approaches to the diagnosis, prognosis, and individualized treatment to modulate the host response to vivax malaria.

Altogether, our data indicate that changes in clinical parameters and biomarkers detected in the plasma of $P$. vivax patients are the result of both systemic host responses and local infection in tissue reservoirs such as BM and spleen. Our analysis shows that measuring a combination of host parameters (e.g. Syndecan-1, IL-6, platelet levels) and total parasite biomass (PvLDH) could predict the extent of parasite infection outside of circulation. Our data also instigate future investigations of systemic signatures with parallel analysis focused on tissue responses, particularly in reservoirs such as the haematopoietic niche of BM and spleen, which has great potential to advance better diagnosis and treatment of $P$. vivax.

\section{Materials and methods}




\begin{tabular}{|c|c|c|c|c|}
\hline $\begin{array}{l}\text { Reagent type (species) } \\
\text { or resource }\end{array}$ & Designation & Source or reference & Identifiers & Additional information \\
\hline Sequence-based reagent & qRT-PCR Oligonucleotides & This study & $\begin{array}{l}\text { See Supplementary } \\
\text { file } 2\end{array}$ & \\
\hline Commercial assay or kit & $\begin{array}{l}\text { Customized multiplex suspension } \\
\text { detection system }\end{array}$ & R\&D Systems & & \\
\hline Commercial assay or kit & $\begin{array}{l}\text { Accutase Cell Detachment } \\
\text { Solution }\end{array}$ & BioLegend & Cat. \#423201 & \\
\hline $\begin{array}{l}\text { Chemical compound, } \\
\text { drug }\end{array}$ & Fixable Viability Dye eFluor 506 & ThermoFisher & Cat. \#65-0866-14 & \\
\hline Software, algorithm & FlowJo software (v10) & Ashland, OR & & https://www.flowjo.com \\
\hline Software, algorithm & RStudio software (v1.4.1106) & RStudio, Boston, MA & & https://www.rstudio.com \\
\hline Software, algorithm & Cytoscape software (v3.8.1) & NIGMS, Bethesda, MD & & https://cytoscape.org \\
\hline Software, algorithm & GraphPad Prism 9 (v9.1.1 (223)) & $\begin{array}{l}\text { GraphPad Software, San Diego, } \\
\text { CA }\end{array}$ & & graphpad.com \\
\hline Software, algorithm & ImageJ software & NIH, Bethesda, MD & & imagej.nih.gov \\
\hline
\end{tabular}

\section{Patients}

Peripheral blood and plasma samples were collected from 79 patients infected with $P$. vivax, as diagnosed by light microscopy, seen at FMT-HVD and 34 healthy donors (controls). Patients and healthy donors were age and sex-matched, with a frequency of $30 \%$ female and $70 \%$ male individuals in both groups. All individuals within the study were from a local vivax malaria epidemic area in the Amazon region of Brazil. All patients included were outpatients that did not meet WHO criteria for severe malaria. Diagnosis was further confirmed by quantitative PCR (qPCR) for both P. vivax and P. falciparum, using previously published nucleotide sequences (RosanasUrgell et al., 2010). Excluding other coinfections could have been of interest. However, the differential diagnosis for an acute febrile illness is very broad and it would be impractical to track all other possible diseases. In addition, the patients included in the present work had mild disease, and therefore were discharged from hospital after a positive malaria diagnosis. No further investigation on other infections was done. The main coinfection to be considered for an acute febrile illness with no localizing signs in our context is dengue fever. Although dengue coinfection in our cohort is possible, the incidence at the hospital is only $2.8 \%(P$. vivax/dengue coinfection; Magalhães et al., 2014Magalhães et al., 2014). Thus, it is unlikely that such a coinfection would have a major impact on our findings. Exclusion criteria were (1) under 18 years of age, (2) pregnancy, (3) use of antimalarials, (4) chronic disease, (5) medication known to interfere with platelet count/function, and (6) smoking.

Anaemia is defined as haemoglobin $<12.5 \mathrm{~g} / \mathrm{dL}$; haematocrit $<37 \%$; RBCs counts $<4.45 \times 10^{6} / \mu \mathrm{L}$. Thrombocytopenia is defined as a decrease in platelet counts to $<150,000 / \mu \mathrm{L}$. Based on platelet levels, patients were grouped into (1) non-thrombocytopenia

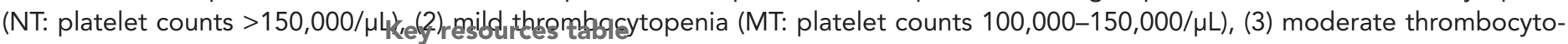
penia (MDT: platelet counts 50,000-100,000/ $\mu \mathrm{L}$ ), and (4) severe thrombocytopenia (ST: platelet counts $<50,000 / \mu \mathrm{L}$ ). Lymphopenia was

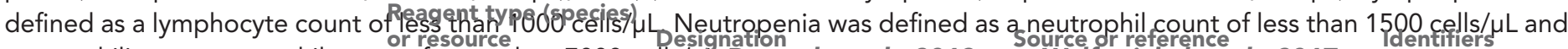

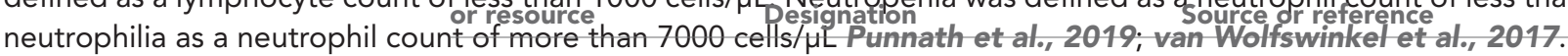

Biological sample (Homo Human umbilical vein endothelial Hematology Center, University of

Preparation of poor platelet plasma cells (HUVEC) Campinas, Campinas, SP, Brazil

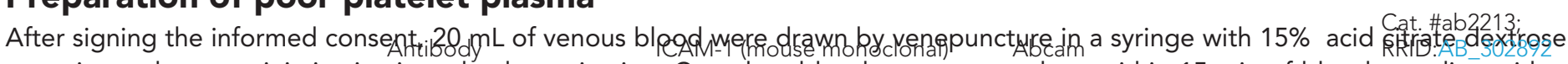
as anticoagulant to minimize in vitro platelet activation. Complete blood counts were done within 15 min of blood sampling with a Sysmex KX21N counter. Whole blood was centrifuged at $180 \mathrm{~g}$ for $18 \mathrm{~min}$ at room temperature, without brake for gradjent formation,

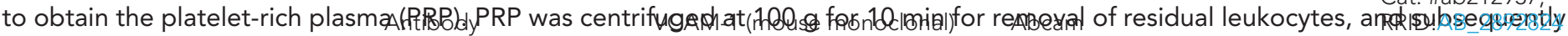
centrifuged at $800 \mathrm{~g}$ for $20 \mathrm{~min}$ to obtain the platelet pellet. Prostaglandin E1 at $300 \mathrm{nM}$ was used to minimize platelet aggregation.

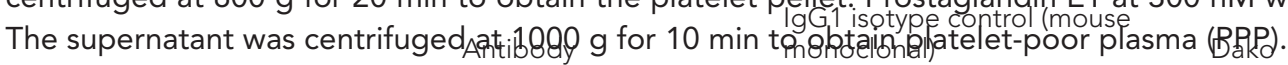

\section{Multiplex bead array assayody ICAM-1 (mouse monoclonal) BioLegend} RRID:AB_2892825 The biomarkers were analysed in thawed plasma with a customized multiplex suspension detection system (R\&D Systems) fer quantification of the following biomarkerfstibody monoclonal) Cat. \#322714; RRID:AB_535986 
(3) Glycocalyx breakdown and EC damage marker: Syndecan-1

(4) Platelet activation markers: CXCL4 and CXCL7

(5) Megakaryocyte differentiation-inducing cytokines: TPO and IL-11; and other proteins such as IL-10, L-selectin, and SCF.

A representative set of $31 \mathrm{P}$. vivax patients were selected for the multiplex assay (Figure 1figure supplement 1). These patients were selected to encompass the wide range of peripheral parasitaemia present in the cohort (260-25,150 infected RBCs/ $\mu \mathrm{L})$ and to match age, gender, and other haematological parameters to those that were not selected. Nine healthy donors matched for age and sex were also selected.

\section{PvLDH ELISA}

To measure PVLDH in patient plasma samples, ELISA was performed using a matching pair of capture and detection antibodies (Vista Diagnostics International LLC, Greenbank, WA). Briefly, 96-well microtiter plate was coated with monoclonal anti-pLDH Vivax-specific (clone 3H8, Vista Diagnostics International LLC; RRID:AB_2892826) at a concentration of $1 \mu \mathrm{g} / \mathrm{mL}$ in PBS (pH 7.4) and incubated overnight at $4{ }^{\circ} \mathrm{C}$. The plate was washed and incubated with blocking buffer (reagent diluent) at room temperature for $1 \mathrm{hr}$. After washing, samples were added and incubated for $2 \mathrm{hr}$. Next, plates were washed and biotinylated anti-PvLDH detection antibody (clone 6c9, Vista Diagnostics International LLC; RRID:AB_2892827), diluted 1:4000 in blocking buffer, was incubated for $2 \mathrm{hr}$ at room temperature, followed by streptavidin-HRP incubation for $20 \mathrm{~min}$ at room temperature. Plates were washed and incubated for $20 \mathrm{~min}$ with substrate solution. Optical density was determined at $450 \mathrm{~nm}$. Cut-off of positivity was defined by correcting absorbance values generated in the plasma samples from healthy donors (controls) by blank values (plate controls), with both values being in the same range. Absorbance values higher than controls were considered positive. In parallel, we used schizont extracts to perform standard curves and lower absorbance values were in the range of O.D $=0.03-0.04$. All positive patient samples gave O.D. values equal to or higher than 0.05 .

\section{PCA and K-means hierarchical clustering}

Haematological parameters (haemoglobin levels, haematocrit, differential blood cell counts), parasite parameters (peripheral parasitaemia by blood smear, parasite load by qPCR, and parasite biomass PvLDH ELISA), and Luminex data (24 biomarkers) from the selected 9 healthy donors and $31 P$. vivax patients were normalized to avoid variable-specific bias and z-score values were determined. Since the host response is complex and multidimensional (one dimension per Luminex biomarker), we applied dimension reduction and clustering for ease of downstream analysis. For this, all variables were used as input for PCA to reduce the dimensionality of data using the PCA function in the FactoMineR package in R. For visualization of PCA results, ggplot2, factoextra, and corrplot packages were used. For each PC, we determined which variables are better represented and the contribution (correlation or loading score) of each variable for each (PC). Investigation of eigenvalues and the percentage of explained variances retained by the PCs demonstrated that the first 10 PCs accounted for the variance of the data (Figure 2-figure supplement 1). However, variables were well represented by the first two PCs (Dim 1 and Dim 2), which were therefore retained for further analysis. In parallel, we performed K-means clustering $(k)$ followed by bootstrapping, which produced the most stable clusters with $k=2$ (cluster $1=21$ individuals; cluster $2=18$ individuals), which seemed to be the most consistent with the data (Figure 2A, Figure 2-figure supplement 1, Figure 2-figure supplement 2, Figure 2-figure supplement 2-source data 1). Figure 2-figure supplement 2-source data 1 contains the numerical data representing cluster stability via bootstrapping. The metrics of interest is jaccard_index, which measures the cluster similarity across bootstrap samples. Similar to the above, $k=2$ gives stable clusters for all configurations (jaccard_index 0.9 and 0.86 ). Using Monte Carlo reference-based consensus clustering (M3C) analysis ( $M 3 C$ function in the $M 3 C$ package in R) indicated that $\mathrm{k}=2$ is the optimal number of clusters when using K-means clustering (Figure 2-figure supplement 2C and D), but when determining spectral clusters, different from elliptical k-means clusters, $k=3$ gives the best number of clusters (Figure 2-figure supplement 2E-G). 


\section{Correlation plots and heatmap visualization}

Heatmaps were created to visualize variable values using R function Complex Heatmap. They represent z-scores using row scaling obtained by centring represented variables with the scale function, followed by column clustering using average cluster method and Euclidean distance metric in R. The same software was used to determine pairwise Pearson's correlation coefficients between variables by running the function cor in the ggcorrplot package and visualized as a correlogram using $\mathrm{R}$ function corrplot in the Hmisc package displaying positive correlations in red and negative correlations in blue using $\mathrm{p} \leq 0.01$ as a cut-off.

\section{Recursive partitioning decision tree classification and machine learning models}

We used recursive partitioning decision tree classification models to evaluate dominant signatures (attributes) predicting a specific outcome. For decision tree construction, we applied the C4.5 algorithm, using the RWeka, caret (Classification and Regression Training) and e1071 packages or the rpart package in R. First, the library caret is used to create a 10-fold training set to train the model. Then, the algorithm implements decision trees (using the $\mathrm{J} 48$ method, which is an open-source Java implementation of the $\mathrm{C} 4.5$ algorithm) starting with all instances in the same group, then repeatedly divides the data based on attributes until each item is classified. The attribute on which to divide is selected by information gain, a statistical technique for determining which attribute split will most cleanly divide the data. To avoid overfitting, sometimes the tree is pruned back. In parallel, the algorithm performs $\mathrm{k}$-fold cross-validation to measure the performance of a given predictive model and indicates which one has the higher accuracy. Here, we used $k=10$ to yield test error rate estimates that suffer neither from excessively high bias nor from very high variance (James et al., 2013). In parallel, features with mean decrease accuracy larger than six were used for random forest. In the random forest analysis, a thousand trees were built using $\mathrm{R}$ package randomForest (version 4.6.14). The normalized additive predicting probability was computed as the final predicting probability. Those selected important features were used for the random forest analysis on the test cohort for model validation.

\section{Stimulation of HUVEC with patients' plasma pools}

After standardization procedures, primary HUVEC were stimulated or not (mock control) in culture media for $6 \mathrm{hr}$ - to evaluate mRNA expression - or for $18 \mathrm{hr}$ - to evaluate protein expression with complete EGM-2 medium (Lonza) containing 30\% (v/v) plasma pools generated from the three subgroups - healthy control, Vivax ${ }^{\text {low }}$ and Vivax ${ }^{\text {high }}$ - and $3 \mathrm{U} / \mathrm{L}$ heparin.

\section{Real-time quantitative RT-PCR}

After $6 \mathrm{hr}$ stimulation, total RNA was isolated from the cell lysate using the miRVana miRNA Extraction kit (Ambion) according to the instructions of the manufacturer. cDNA was synthesized with TaqMan Reverse Transcriptase (Applied Biosystems, Foster City, CA) and mRNA expression of genes were determined by qRT-PCR. Real-time qRT-PCR was performed on an ABI-Prism 7000 PCR cycler (Applied Biosystems) or on the CFX96 Real-Time PCR Detection System (Bio-Rad). Cycling parameters were $95^{\circ} \mathrm{C}$ for $1 \mathrm{~min}$ and then 35 cycles of $95^{\circ} \mathrm{C}(15 \mathrm{~s})$ and $60{ }^{\circ} \mathrm{C}(1 \mathrm{~min})$, followed by a melting curve analysis. The median cycle threshold $\left(C_{t}\right)$ value and $2^{-\Delta \Delta C t}$ method were used for relative quantification analysis, and all $C_{t}$ values were normalized to the GAPDH mRNA expression level. Results expressed as means and SEM of biological replicates are shown. The mock sample (HUVECs incubated with culture media only) was used as reference. The oligonucleotides used are described in Supplementary file 2.

\section{Endothelial cell flow cytometry (FC) and immunofluorescence analysis (IFA)}

For IFA, cells were grown in eight-well chambered coverslips (IBIDI) until confluence. After $18 \mathrm{hr}$ stimulation with plasma pools, cells were washed with PBS and fixed/permeabilized with ice-cold $100 \%$ methanol for $5 \mathrm{~min}$ at $-20{ }^{\circ} \mathrm{C}$. In brief, cells were incubated with $10 \%$ goat serum (ThermoFisher) to avoid secondary antibody nonspecific binding for $1 \mathrm{hr}$ at room temperature and then incubated with specific primary antibodies to human ICAM-1 (mouse monoclonal clone MEM-111; Abcam; Cat. \# 
ab2213; RRID: AB_302892; used at a dilution of 1:100 in 10\% goat serum); VCAM-1 (mouse monoclonal clone 1.4C3; Abcam; Cat. \# ab212937; RRID: AB_2892824; used at a dilution of 1:500 in 10\% goat serum); and mouse lgG1 isotype control (Dako; Cat. \# X0931; RRID: AB_2892825; used at a dilution of $1: 10$ in $10 \%$ goat serum) overnight at $4{ }^{\circ} \mathrm{C}$. After washing, wells were overlaid for $1 \mathrm{hr}$ with AF488-conjugated secondary antibody (used at a dilution of 1:500 in 10\% goat serum) and Hoechst (diluted at 1:2000) at room temperature. For controls, primary antibodies were omitted from the staining procedure and were negative for any reactivity. The chambers were placed at $4{ }^{\circ} \mathrm{C}$ until use for immunofluorescence assay (IFA). Percentage of positive cells and expression profiles for ICAM1 and VCAM-1 were then determined using ImageJ software (NIH, Bethesda, MD).

In flow cytometry, after $18 \mathrm{hr}$ stimulation with $30 \%$ plasma pools, cells were washed $2 \times$ with DPBS and treated with Accutase Cell Detachment Solution (BioLegend) at room temperature for up to $3 \mathrm{~min}$ or until the cells are detached. Cell count and viability with trypan blue dye were determined and cells were resuspended in ice-cold DPBS without calcium/magnesium, $0.5 \mathrm{mM}$ EDTA, and 10\% foetal bovine serum (FBS; Gibco). Cells were incubated with FcBlock (BD Biosciences, San Jose, CA), followed by incubation with unconjugated anti-VCAM (mouse monoclonal clone 1.4C3; Abcam; Cat. \# ab212937) or AF488-conjugated anti-ICAM-1 (mouse monoclonal clone HCD54; BioLegend; Cat. \# 322714; RRID:AB_535986) or unconjugated mouse lgG1 isotype control (Dako; Cat. \# X0931) for $1 \mathrm{hr}$ at $4{ }^{\circ} \mathrm{C}$. Cells were then washed and incubated for $1 \mathrm{hr}$ at $4{ }^{\circ} \mathrm{C}$ with secondary antibody AF488conjugated anti-mouse IgG (ThermoFisher). Finally, cells were incubated with Fixable Viability Dye eFluor 506 (ThermoFisher) in DPBS without calcium/magnesium, $0.5 \mathrm{mM}$ EDTA for $30 \mathrm{~min}$ at $4{ }^{\circ} \mathrm{C}$. Cells were washed and resuspended in buffer and acquired using a BD FACSCelesta cytometer $(100,000$ events/sample). Percentage of positive cells and expression profiles for ICAM-1 and VCAM-1 were then determined by the mean fluorescence intensity using FlowJo software (v10; Ashland, OR).

\section{Ex vivo evaluation of endothelial cell monolayer function}

EC monolayer function was measured using ECIS, an electric cell-substrate impedance sensing system (ECIS ZO, Applied Biophysics, Troy, NY), as previously described (Santaterra et al., 2020). The system then applies weak alternating currents through the electrode array and continuously measures the ability of the cell monolayer to impede the movement of electrons between adjacent EC (resistance). Briefly, cells were seeded at $2.5 \times 10^{5}$ cells/well on fibronectin-coated $(10 \mu \mathrm{g} / \mathrm{mL})$ eight-well arrays (8WE10, Applied Biophysics) containing interdigitated gold electrodes. ECs were seeded $48 \mathrm{hr}$ before experiments and the resistance started to be recorded after $48 \mathrm{hr}$. Only wells with resistance $>1500$ ohms and stable impedance/resistance readings were used. Before stimulation, resistance was continuously monitored for $2 \mathrm{hr}$ to confirm monolayer stability represented by a plateau in the resistance curve. Stimuli ( $20 \% \mathrm{v} / \mathrm{v}$ pooled plasma in complete medium) was then added to wells under continuous impedance/resistance monitoring for $12 \mathrm{hr}$. A baseline resistance value was recorded immediately prior to the addition of each stimuli, and results are expressed as a ratio from baseline resistance (normalized resistance).

\section{Network analysis}

The values of each circulating factor measured in the plasma samples, as well as haematological parameters and parasite biomass in healthy donors and $P$. vivax malaria patients, were input in the RStudio software (version 1.4.1106, 2021) to determine pair-wise Pearson's correlation coefficients to generate correlation networks and the $\mathrm{p}$-value to test for non-correlation was evaluated using $\mathrm{p} \leq 0.05$ as a cut-off. In order to analyse the structure of the networks, edges list was generated in $\mathrm{R}$ using the functions melt (reshape2 package), graph_from_edgelist (igraph package). Graphs were customized in the Cytoscape software (version 3.8.1) using the force-directed layout, which simulates a system of forces, determined by the correlation strength. In the equilibrium state, edges tend to have uniform length, and nodes that are not connected by an edge tend to be drawn further apart. Network topology and module analysis were performed using the NetworkAnalyzer, jActiveModules, and MCODE plugins in Cytoscape (Cline et al., 2007; Doncheva et al., 2012). Supplementary file 1 shows the results for all parameters quantified in the comparative network topology analysis between the graphs for healthy donors and $P$. vivax patients. 


\section{Statistical analysis}

Fisher's exact test was used for categorical data. Data normality was checked by Shapiro-Wilk test. Student's t-test was used to compare means between groups with normally distributed data, and data sets with non-normal distributions were compared using Mann-Whitney test. All tests were performed two-sided using a nominal significance threshold of $p<0.05$ unless otherwise specified. When appropriate to adjust for multiple hypothesis testing, Tukey's or Bonferroni corrected multiple comparisons test significance at the $p$-value $<0.05$ threshold was performed unless otherwise specified. Data are presented as scatter plots with median and 25-75\% interquartile range, box plots showing minimum to maximum range or means and SEM, unless otherwise stated. Analyses were performed and the graphs generated in GraphPad Prism 9 (version 9.1.1 [223], 2021) and RStudio software (version 1.4.1106; 2021). To ensure that differences observed between $P$. vivax- infected patients and controls, as well as between the clusters, were due to disease status and not confounded by age or sex, the clinical parameters were fitted as response variables in a linear model with sex and/or age fitted as explanatory variables. Age and sex were included in the model if their coefficients were estimated as different from zero with $\mathrm{p}$-value $<0.05$. The residuals from the linear model were then used as ageand/or sex-corrected parameters in subsequent analyses.

\section{Study approval}

All subjects enrolled in the study were adults. Written informed consent was obtained from all participants, and the study was conducted according to the Declaration of Helsinki principles. The study was approved by the local Research Ethics Committee at Fundação de Medicina Tropical Dr. Heitor Vieira Dourado (FMT-HVD, Manaus, Brazil), under \#CAAE: 54234216.1.0000.0005.

\section{Acknowledgements}

We would like to thank all patients enrolled in this research and the support of the malaria diagnosis and field team at field team in the Fundação de Medicina Tropical Dr. Heitor Vieira Dourado (FMTHVD) in Manaus, Brazil. The authors also gratefully acknowledge the help and assistance provided by the Central Laboratory of High-Performance Technologies (LaCTAD, University of Campinas) and the Institute of Infection, Immunity and Inflammation Flow Core Facility in the generation of some of the data reported in this manuscript. MM was supported by a Wolfson Merit Award from the Royal Society and Wellcome Trust Center award (number 104111). JLSF was supported by the Sao Paulo Research Foundation (FAPESP grant 2019/01578-2 and 2016/12855-9), and FTMC was supported by the Sao Paulo Research Foundation (FAPESP grant 2017/18611-7). MVGL and FTMC are CNPq research fellows.

\section{Additional information}

\begin{tabular}{lll}
$\begin{array}{l}\text { Funding } \\
\text { Funder }\end{array}$ & Grant reference number & Author \\
\hline $\begin{array}{l}\text { Fundação de Amparo à } \\
\begin{array}{l}\text { Pesquisa do Estado de São } \\
\text { Paulo }\end{array}\end{array}$ & João L Silva-Filho \\
$\begin{array}{ll}\text { Fundação de Amparo à } \\
\begin{array}{l}\text { Pesquisa do Estado de São } \\
\text { Paulo }\end{array}\end{array}$ & Fabio TM Costa \\
\hline $\begin{array}{l}\text { Wellcome Trust } \\
\begin{array}{l}\text { Fundação de Amparo à } \\
\text { Pesquisa do Estado de São }\end{array}\end{array}$ & Matthias Marti \\
\begin{tabular}{l} 
Paulo \\
\hline
\end{tabular}
\end{tabular}

The funders had no role in study design, data collection and interpretation, or the decision to submit the work for publication. 
Author contributions

João L Silva-Filho, João CK Dos-Santos, Conceptualization, Data curation, Formal analysis, Investigation, Methodology, Project administration, Supervision, Validation, Visualization, Writing - original draft, Writing - review and editing; Carla Judice, Conceptualization, Data curation, Formal analysis, Investigation, Methodology, Validation; Dario Beraldi, Data curation, Formal analysis, Methodology, Software, Validation, Writing - review and editing; Kannan Venugopal, Formal analysis, Investigation, Methodology; Diogenes Lima, Data curation, Formal analysis, Investigation, Supervision, Validation; Helder I Nakaya, Conceptualization, Formal analysis, Supervision, Validation; Erich V De Paula, Conceptualization, Data curation, Formal analysis, Investigation, Methodology, Supervision, Validation, Writing - review and editing; Stefanie CP Lopes, Conceptualization, Data curation, Formal analysis, Funding acquisition, Investigation, Methodology, Supervision, Validation, Visualization, Writing - review and editing; Marcus VG Lacerda, Conceptualization, Data curation, Funding acquisition, Project administration, Supervision, Validation, Writing - review and editing; Matthias Marti, Formal analysis, Funding acquisition, Investigation, Methodology, Project administration, Resources, Supervision, Validation, Visualization, Writing - original draft, Writing - review and editing; Fabio TM Costa, Conceptualization, Data curation, Funding acquisition, Investigation, Methodology, Project administration, Resources, Supervision, Validation, Visualization, Writing - original draft, Writing - review and editing

\section{Author ORCIDs}

João L Silva-Filho (iD http://orcid.org/0000-0003-4762-2205

João CK Dos-Santos (iD http://orcid.org/0000-0001-5916-9845

Carla Judice (iD http://orcid.org/0000-0003-1839-053X

Helder I Nakaya (iD http://orcid.org/0000-0001-5297-9108

Matthias Marti (iD http://orcid.org/0000-0003-1040-9566

Fabio TM Costa (id http://orcid.org/0000-0001-9969-7300

Ethics

All subjects enrolled in the study were adults. Written informed consent was obtained from all participants and the study was conducted according to the Declaration of Helsinki principles. The study was approved by the local Research Ethics Committee at Fundação de Medicina Tropical Dr. Heitor Vieira Dourado (FMT-HVD, Manaus, Brazil), under \#CAAE: 54234216.1.0000.0005 and by the Research Ethics Committee at University of Campinas (UNICAMP, Campinas, Brazil), under \#CAAE: 54234216.1.3001.5404.

Decision letter and Author response

Decision letter https://doi.org/10.7554/eLife.71351.sa1

Author response https://doi.org/10.7554/eLife.71351.sa2

\section{Additional files}

Supplementary files

- Supplementary file 1. Topological analysis of the network graphs of healthy donors and P. vivax patients.

- Supplementary file 2. Oligonucleotides sequences used in the qRT-PCRs.

- Transparent reporting form

Data availability

All data generated or analysed during this study are included in the manuscript and supporting files. Numerical tables and source data files have been provided. Table 1, Figure 2-source data 1 and Figure 2-figure supplement 2-source data 1 contain the numerical data used to generate the figures. 


\section{References}

Andrade BB, Reis-Filho A, Souza-Neto SM. 2010. Severe Plasmodium vivax malaria exhibits marked inflammatory imbalance. Malaria Journal 9: 13. DOI: https://doi.org/10.1186/1475-2875-9-13, PMID: 20070895

Anstey NM, Jacups SP, Cain T. 2002. Pulmonary manifestations of uncomplicated falciparum and vivax malaria: cough, small airways obstruction, impaired gas transfer, and increased pulmonary phagocytic activity. The Journal of Infectious Diseases 185: 1326-1334. DOI: https://doi.org/10.1086/339885, PMID: 12001051

Anstey NM, Handojo T, Pain MC. 2007. Lung injury in vivax malaria: pathophysiological evidence for pulmonary vascular sequestration and posttreatment alveolar-capillary inflammation. The Journal of Infectious Diseases 195: 589-596. DOI: https://doi.org/10.1086/510756, PMID: 17230420

Baird JK. 2013. Evidence and implications of mortality associated with acute Plasmodium vivax malaria. Clinical Microbiology Reviews 26: 36-57. DOI: https://doi.org/10.1128/CMR.00074-12, PMID: 23297258

Barber BE, William T, Grigg MJ. 2015. Parasite biomass-related inflammation, endothelial activation, microvascular dysfunction and disease severity in vivax malaria. PLOS Pathogens 11: e1004558. DOI: https:// doi.org/10.1371/journal.ppat.1004558, PMID: 25569250

Barber BE, Grigg MJ, Piera KA. 2021. Endothelial glycocalyx degradation and disease severity in Plasmodium vivax and Plasmodium knowlesi malaria. Scientific Reports 11: 9741. DOI: https://doi.org/10.1038/s41598-02188962-6, PMID: 33963210

Baro B, Deroost K, Raiol T. 2017. Plasmodium vivax gametocytes in the bone marrow of an acute malaria patient and changes in the erythroid miRNA profile. PLOS Neglected Tropical Diseases 11: e0005365. DOI: https://doi. org/10.1371/journal.pntd.0005365, PMID: 28384192

Becker BF, Jacob M, Leipert S, Salmon AH, Chappell D. 2015. Degradation of the endothelial glycocalyx in clinical settings: searching for the sheddases. British Journal of Clinical Pharmacology 80: 389-402. DOI: https://doi.org/10.1111/bcp.12629, PMID: 25778676

Bernardo A, Ball C, Nolasco L, Moake JF, Dong JF. 2004. Effects of inflammatory cytokines on the release and cleavage of the endothelial cell-derived ultralarge von Willebrand factor multimers under flow. Blood 104: 100-106. DOI: https://doi.org/10.1182/blood-2004-01-0107, PMID: 15026315

Bevilacqua MP. 1993. Endothelial-leukocyte adhesion molecules. Annual Review of Immunology 11: 767-804 DOI: https://doi.org/10.1146/annurev.iy.11.040193.004003, PMID: 8476577

Boiko JR, Borghesi L. 2012. Hematopoiesis sculpted by pathogens: Toll-like receptors and inflammatory mediators directly activate stem cells. Cytokine 57: 1-8. DOI: https://doi.org/10.1016/j.cyto.2011.10.005, PMID: 22079335

Brito MAM, Baro B, Raiol TC. 2020. Morphological and Transcriptional Changes in Human Bone Marrow During Natural Plasmodium vivax Malaria Infections. The Journal of Infectious Diseases 10: jiaa177. DOI: https://doi. org/10.1093/infdis/jiaa177, PMID: 32556188

Carvalho BO, Lopes SC, Nogueira PA. 2010. On the cytoadhesion of Plasmodium vivax-infected erythrocytes. The Journal of Infectious Diseases 202: 638-647. DOI: https://doi.org/10.1086/654815, PMID: 20617923

Chiba Y, Mizoguchi I, Hasegawa H. 2018. Regulation of myelopoiesis by proinflammatory cytokines in infectious diseases. Cellular and Molecular Life Sciences 75: 1363-1376. DOI: https://doi.org/10.1007/s00018-017-27245, PMID: 29218601

Cline MS, Smoot M, Cerami E. 2007. Integration of biological networks and gene expression data using Cytoscape. Nature Protocols 2: 2366-2382. DOI: https://doi.org/10.1038/nprot.2007.324, PMID: 17947979

Crockett-Torabi E, Sulenbarger B, Smith CW, Fantone JC. 1995. Activation of human neutrophils through L-selectin and Mac-1 molecules. Journal of Immunology 154: 2291-2302 PMID: $7532664 .$,

De las Salas B, Segura C, Pabón A, Lopes SC, Costa FT, Blair S. 2013. Adherence to human lung microvascular endothelial cells (HMVEC-L) of Plasmodium vivax isolates from Colombia. Malaria Journal 12: 347. DOI: https:// doi.org/10.1186/1475-2875-12-347, PMID: 24080027

de Mast Q, Groot E, Lenting PJ. 2007. Thrombocytopenia and release of activated von Willebrand Factor during early Plasmodium falciparum malaria. The Journal of Infectious Diseases 196: 622-628. DOI: https://doi.org/10. 1086/519844, PMID: 17624850

de Mast Q, Groot E, Asih PB. 2009. ADAMTS13 deficiency with elevated levels of ultra-large and active von Willebrand factor in P. Falciparum and P. Vivax Malaria. Am J Trop Med Hyg 80: 492-498 PMID: 19270304., Doncheva NT, Assenov Y, Domingues FS, Albrecht M. 2012. Topological analysis and interactive visualization of biological networks and protein structures. Nature Protocols 7: 670-685. DOI: https://doi.org/10.1038/nprot. 2012.004, PMID: 22422314

Dos-Santos JCK, Silva-Filho JL, Judice CC. 2020. Platelet disturbances correlate with endothelial cell activation in uncomplicated Plasmodium vivax malaria. PLOS Neglected Tropical Diseases 14: e0007656. DOI: https:// doi.org/10.1371/journal.pntd.0007656, PMID: 32687542

Druilhe P, Brasseur P, Blanc C, Makler M. 2007. Improved assessment of plasmodium vivax response to antimalarial drugs by a colorimetric double-site plasmodium lactate dehydrogenase antigen capture enzymelinked immunosorbent assay. Antimicrobial Agents and Chemotherapy 51: 2112-2116. DOI: https://doi.org/10. 1128/AAC.01385-06, PMID: 17387158

Fonseca LL, Joyner CJ, Galinski MR, Voit EO, Consortium M. 2017. A model of Plasmodium vivax concealment based on Plasmodium cynomolgi infections in Macaca mulatta. Malaria Journal 16: 375. DOI: https://doi.org/ 10.1186/s12936-017-2008-4, PMID: 28923058 
Frankenstein Z, Alon U, Cohen IR. 2006. The immune-body cytokine network defines a social architecture of cell interactions. Biology Direct 1: 32. DOI: https://doi.org/10.1186/1745-6150-1-32, PMID: 17062134

Gerardin P, Rogier C, As K, Jouvencel P, Brousse V, Imbert P. 2002. Prognostic value of thrombocytopenia in African children with falciparum malaria. The American Journal of Tropical Medicine and Hygiene 66: 686-691. DOI: https://doi.org/10.4269/ajtmh.2002.66.686, PMID: 12224575

Gomes LT, Alves-Junior ER, Rodrigues-Jesus C, Nery AF, Gasquez-Martin TO, Fontes CJ. 2014. Angiopoietin-2 and angiopoietin-2/angiopoietin-1 ratio as indicators of potential severity of plasmodium vivax malaria in patients with thrombocytopenia. PLOS ONE 9: e109246. DOI: https://doi.org/10.1371/ journal.pone.0109246

Graham SM, Chen J, Chung DW. 2016. Endothelial activation, haemostasis and thrombosis biomarkers in Ugandan children with severe malaria participating in a clinical trial. Malaria Journal 15: 56. DOI: https://doi. org/10.1186/s12936-016-1106-z, PMID: 26830467

Hempel C, Wang CW, Kurtzhals JAL, Staalso T. 2017. Binding of Plasmodium falciparum to CD36 can be shielded by the glycocalyx. Malaria Journal 16: 193. DOI: https://doi.org/10.1186/s12936-017-1844-6, PMID: 28486940

Hviid L, Kemp K. 2000. What is the cause of lymphopenia in malaria? Infection and Immunity 68: 6087-6089. DOI: https://doi.org/10.1128/IAI.68.10.6087-6089.2000, PMID: 11203040

Introini V, Carciati A, Tomaiuolo G, Cicuta P, Guido S. 2018. Endothelial glycocalyx regulates cytoadherence in Plasmodium falciparum malaria. Journal of the Royal Society, Interface 15: 149. DOI: https://doi.org/10.1098/ rsif.2018.0773

Ivetic A. 2018. A head-to-tail view of L-selectin and its impact on neutrophil behaviour. Cell and Tissue Research 371: 437-453. DOI: https://doi.org/10.1007/s00441-017-2774-x, PMID: 29353325

James G, Witten D, Hastie T, Tibshirani R. 2013. An introduction to statistical learning with applications in R introduction. Springer Texts Stat 103: 1-14.

Kho S, Qotrunnada L, Leonardo L. 2021a. Evaluation of splenic accumulation and colocalization of immature reticulocytes and Plasmodium vivax in asymptomatic malaria: A prospective human splenectomy study. PLOS Medicine 18: e1003632. DOI: https://doi.org/10.1371/journal.pmed.1003632, PMID: 34038413

Kho S, Qotrunnada L, Leonardo L. 2021b. Hidden Biomass of Intact Malaria Parasites in the Human Spleen. The New England Journal of Medicine 384: 2067-2069. DOI: https://doi.org/10.1056/NEJMc2023884, PMID: 34042394

Klei TR, Meinderts SM, van den Berg TK, van Bruggen R. 2017. From the Cradle to the Grave: The Role of Macrophages in Erythropoiesis and Erythrophagocytosis. Frontiers in Immunology 8: 73. DOI: https://doi.org/ 10.3389/fimmu.2017.00073, PMID: 28210260

Kochar DK, Saxena V, Singh N, Kochar SK, Kumar SV, Das A. 2005. Plasmodium vivax malaria. Emerg Infect Dis 11: 132-134. DOI: https://doi.org/10.3201/eid1101.040519, PMID: 15705338

Kochar DK, Das A, Kochar A. 2010. Thrombocytopenia in Plasmodium falciparum, Plasmodium vivax and mixed infection malaria: a study from Bikaner (Northwestern India). Platelets 21: 623-627. DOI: https://doi.org/10. 3109/09537104.2010.505308, PMID: 21050055

Kovtonyuk LV, Fritsch K, Feng X, Manz MG, Takizawa H. 2016. Inflamm-Aging of Hematopoiesis, Hematopoietic Stem Cells, and the Bone Marrow Microenvironment. Frontiers in Immunology 7: 502. DOI: https://doi.org/10. 3389/fimmu.2016.00502, PMID: 27895645

Lacerda MV, Hipólito JR, Passos LN. 2008. Chronic Plasmodium vivax infection in a patient with splenomegaly and severe thrombocytopenia. Revista Da Sociedade Brasileira de Medicina Tropical 41: 522-523. DOI: https:// doi.org/10.1590/s0037-86822008000500021, PMID: 19009202

Lacerda MV, Mourao MP, Coelho HC, Santos JB. 2011. Thrombocytopenia in malaria: who cares? Memorias Do Instituto Oswaldo Cruz 106: 52-63. DOI: https://doi.org/10.1590/s0074-02762011000900007, PMID: 21881757

Lacerda MV, Fragoso SC, Alecrim MG. 2012. Postmortem characterization of patients with clinical diagnosis of Plasmodium vivax malaria: to what extent does this parasite kill? Clinical Infectious Diseases 55: 67-74. DOI: https://doi.org/10.1093/cid/cis615, PMID: 22772803

Lazzari E, Butler JM. 2018. The Instructive Role of the Bone Marrow Niche in Aging and Leukemia. Current Stem Cell Reports 4: 291-298. DOI: https://doi.org/10.1007/s40778-018-0143-7, PMID: 30524938

Lopes SC, Albrecht L, Carvalho BO. 2014. Paucity of Plasmodium vivax mature schizonts in peripheral blood is associated with their increased cytoadhesive potential. The Journal of Infectious Diseases 209: 1403-1407. DOI: https://doi.org/10.1093/infdis/jiu018, PMID: 24415786

Machado Siqueira A, Lopes Magalhães BM, Cardoso Melo G. 2012. Spleen rupture in a case of untreated Plasmodium vivax infection. PLOS Neglected Tropical Diseases 6: : e1934. DOI: https://doi.org/10.1371/ journal.pntd.0001934

Magalhães BML, Siqueira AM, Alexandre MAA, Souza MS, Gimaque JB, Bastos MS, Figueiredo RMP, Melo GC, Lacerda MVG, Mourão MPG. 2014. P. Vivax malaria and Dengue fever co-infection: A cross-sectional study in the brazilian Amazon. PLOS Neglected Tropical Diseases 8: e3239. DOI: https://doi.org/10.1371/journal.pntd. 0003239, PMID: 25340346

Malleret B, Li A, Zhang R. 2015. Plasmodium vivax: restricted tropism and rapid remodeling of CD71-positive reticulocytes. Blood 125: 1314-1324. DOI: https://doi.org/10.1182/blood-2014-08-596015, PMID: 25414440

Mantel PY, Hoang AN, Goldowitz I. 2013. Malaria-infected erythrocyte-derived microvesicles mediate cellular communication within the parasite population and with the host immune system. Cell Host \& Microbe 13: 521-534. DOI: https://doi.org/10.1016/j.chom.2013.04.009, PMID: 23684304 
Mantel PY, Hjelmqvist D, Walch M. 2016. Infected erythrocyte-derived extracellular vesicles alter vascular function via regulatory Ago2-miRNA complexes in malaria. Nature Communications 7: 12727. DOI: https://doi. org/10.1038/ncomms12727, PMID: 27721445

Mayor A, Alano P. 2015. Bone marrow reticulocytes: a Plasmodium vivax affair. Blood 125: 1203-1205. DOI: https://doi.org/10.1182/blood-2014-12-614123, PMID: 25700424

Mendonça VR, Queiroz AT, Lopes FM, Andrade BB, Barral-Netto M. 2013. Networking the host immune response in Plasmodium vivax malaria. Malaria Journal 12: 69. DOI: https://doi.org/10.1186/1475-2875-12-69, PMID: 23433077

Naing C, Whittaker MA. 2018. Severe thrombocytopaenia in patients with vivax malaria compared to falciparum malaria: a systematic review and meta-analysis. Infectious Diseases of Poverty 7: 10. DOI: https://doi.org/10. 1186/s40249-018-0392-9, PMID: 29427995

Obaldia N, Meibalan E, Jm S. 2018. Bone Marrow Is a Major Parasite Reservoir in Plasmodium vivax Infection. MBio 9: e00625-18. DOI: https://doi.org/10.1128/mBio.00625-18, PMID: 29739900

O’Donnell J, Mille-Baker B, Laffan M. 2000. Human umbilical vein endothelial cells differ from other endothelial cells in failing to express ABO blood group antigens. Journal of Vascular Research 37: 540-547. DOI: https:// doi.org/10.1159/000054087, PMID: 11146408

Park JW, Park SH, Yeom JS. 2003. Serum cytokine profiles in patients with Plasmodium vivax malaria: a comparison between those who presented with and without thrombocytopenia. Annals of Tropical Medicine and Parasitology 97: 339-344. DOI: https://doi.org/10.1179/000349803235002416, PMID: 12831519

Pillinger NL, Kam P. 2017. Endothelial glycocalyx: basic science and clinical implications. Anaesthesia and Intensive Care 10: 295-307. DOI: https://doi.org/10.1177/0310057X1704500305, PMID: 28486888

Punnath K, Dayanand KK, Chandrashekar VN. 2019. Association between Inflammatory Cytokine Levels and Thrombocytopenia during Plasmodium falciparum and P. vivax Infections in South-Western Coastal Region of India. Malaria Research and Treatment 2019: 4296523. DOI: https://doi.org/10.1155/2019/4296523, PMID: 31110658

Rhodes MM, Koury ST, Kopsombut P, Alford CE, Price JO, Koury MJ. 2016. Stress reticulocytes lose transferrin receptors by an extrinsic process involving spleen and macrophages. American Journal of Hematology 91 : 875-882. DOI: https://doi.org/10.1002/ajh.24421, PMID: 27194638

Rodriguez-Morales AJ, Sanchez E, Arria M. 2005. White blood cell counts in Plasmodium vivax malaria. The Journal of Infectious Diseases 192: 1675-1676. DOI: https://doi.org/10.1086/496993, PMID: 16206089

Rosanas-Urgell A, Mueller D, Betuela I. 2010. Comparison of diagnostic methods for the detection and quantification of the four sympatric Plasmodium species in field samples from Papua New Guinea. Malaria Journal 9: 361. DOI: https://doi.org/10.1186/1475-2875-9-361, PMID: 21156052

Salutari P, Sica S, Chiusolo P. 1996. Plasmodium vivax malaria after autologous bone marrow transplantation: an unusual complication. Bone Marrow Transplantation 18: 805-806 PMID: $8899200 .$,

Santaterra VAG, Fiusa MML, Hounkpe BW. 2020. Endothelial Barrier Integrity Is Disrupted In Vitro by Heme and by Serum From Sickle Cell Disease Patients. Frontiers in Immunology 11: 535147. DOI: https://doi.org/10. 3389/fimmu.2020.535147, PMID: 33381108

Silva-Filho JL, Recker M, Wassmer SC, Costa FTM. 2021. Plasmodium vivax in hematopoietic niches: Hidden and dangerous2020. Trends in Parasitology 36: 648-649. DOI: https://doi.org/10.1016/j.pt.2020.05.006

Soehnlein O, Steffens S, Hidalgo A, Weber C. 2017. Neutrophils as protagonists and targets in chronic inflammation. Nature Reviews. Immunology 17: 248-261. DOI: https://doi.org/10.1038/nri.2017.10, PMID: 28287106

Tan PH, Chan C, Xue SA. 2004. Phenotypic and functional differences between human saphenous vein (HSVEC) and umbilical vein (HUVEC) endothelial cells. Atherosclerosis 173: 171-183. DOI: https://doi.org/10.1016/j. atherosclerosis.2003.12.011, PMID: 15064090

Tan LK, Yacoub S, Scott S, Bhagani S, Jacobs M. 2008. Acute lung injury and other serious complications of Plasmodium vivax malaria. The Lancet. Infectious Diseases 8: 449-454. DOI: https://doi.org/10.1016/S14733099(08)70153-1, PMID: 18582837

Tangpukdee N, Yew HS, Krudsood S. 2008. Dynamic changes in white blood cell counts in uncomplicated Plasmodium falciparum and P. vivax malaria. Parasitology International 57: 490-494. DOI: https://doi.org/10. 1016/j.parint.2008.06.005, PMID: 18647661

Toda H, Diaz-Varela M, Segui-Barber J. 2020. Plasma-derived extracellular vesicles from Plasmodium vivax patients signal spleen fibroblasts via NF-kB facilitating parasite cytoadherence. Nature Communications 11: 2761. DOI: https://doi.org/10.1038/s41467-020-16337-y, PMID: 32487994

Val F, Machado K, Barbosa L. 2017. Respiratory Complications of Plasmodium vivax Malaria: Systematic Review and Meta-Analysis. The American Journal of Tropical Medicine and Hygiene 97: 733-743. DOI: https://doi.org/ 10.4269/ajtmh.17-0131, PMID: 28722625

Valecha N, Pinto RG, Turner GD. 2009. Histopathology of fatal respiratory distress caused by Plasmodium vivax malaria. The American Journal of Tropical Medicine and Hygiene 81: 758-762. DOI: https://doi.org/10.4269/ ajtmh.2009.09-0348, PMID: 19861606

van Wolfswinkel ME, Langenberg MCC, Wammes LJ. 2017. Changes in total and differential leukocyte counts during the clinically silent liver phase in a controlled human malaria infection in malaria-naive Dutch volunteers. Malaria Journal 16: 457. DOI: https://doi.org/10.1186/s12936-017-2108-1, PMID: 29126422

Wickramasinghe SN, Looareesuwan S, Nagachinta B, White NJ. 1989. Dyserythropoiesis and ineffective erythropoiesis in Plasmodium vivax malaria. British Journal of Haematology 72: 91-99. DOI: https://doi.org/10. 1111/j.1365-2141.1989.tb07658.x, PMID: 2660903 
Wickramasinghe SN, Abdalla SH. 2000. Blood and bone marrow changes in malaria. Bailliere's Best Practice \& Research. Clinical Haematology 13: 277-299. DOI: https://doi.org/10.1053/beha.1999.0072, PMID: 10942626 World Malaria Reports. 2019. World Malaria Reports. World Malaria Reports.

Yeo TW, Lampah DA, Tjitra E. 2010. Greater endothelial activation, Weibel-Palade body release and host inflammatory response to Plasmodium vivax, compared with Plasmodium falciparum: a prospective study in Papua, Indonesia. The Journal of Infectious Diseases 202: 109-112. DOI: https://doi.org/10.1086/653211, PMID: 20497057

Yeo TW, Weinberg JB, Lampah DA. 2019. Glycocalyx Breakdown Is Associated With Severe Disease and Fatal Outcome in Plasmodium falciparum Malaria. Clinical Infectious Diseases 69: 1712-1720. DOI: https://doi.org/ 10.1093/cid/ciz038, PMID: 30753363

Yx R, Mao BY, Zhang FK. 2009. Invasion of erythroblasts by Pasmodium vivax: A new mechanism contributing to malarial anemia. Ultrastructural Pathology 33: 236-242. DOI: https://doi.org/10.3109/01913120903251643, PMID: 19895296 